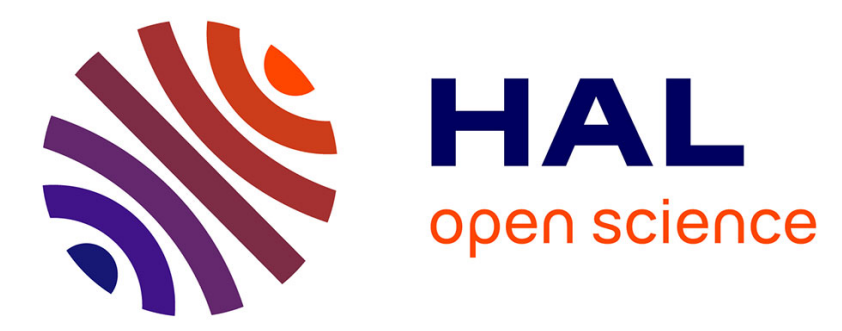

\title{
Acceleration of finite-time stable homogeneous systems
}

Yotam Dvir, Arie Levant, Denis Efimov, Andrey Polyakov, Wilfrid Perruquetti

\section{To cite this version:}

Yotam Dvir, Arie Levant, Denis Efimov, Andrey Polyakov, Wilfrid Perruquetti. Acceleration of finitetime stable homogeneous systems. International Journal of Robust and Nonlinear Control, 2018, 00, pp.1 - 23. 10.1002/rnc . hal-01611961

\section{HAL Id: hal-01611961 \\ https://hal.inria.fr/hal-01611961}

Submitted on 6 Oct 2017

HAL is a multi-disciplinary open access archive for the deposit and dissemination of scientific research documents, whether they are published or not. The documents may come from teaching and research institutions in France or abroad, or from public or private research centers.
L'archive ouverte pluridisciplinaire $\mathbf{H A L}$, est destinée au dépôt et à la diffusion de documents scientifiques de niveau recherche, publiés ou non, émanant des établissements d'enseignement et de recherche français ou étrangers, des laboratoires publics ou privés. 


\title{
Acceleration of finite-time stable homogeneous systems
}

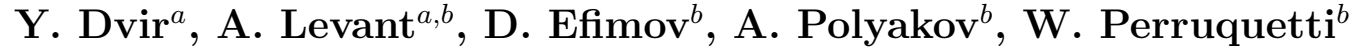 \\ a - School of Mathematical Sciences, Tel-Aviv University, Tel-Aviv 6997801, Israel \\ b-Non-A team, Inria Lille-Nord Europe, Parc Scientifique de la Haute Borne, 40, avenue Halley, Bat. A, \\ Park Plaza, 59650 Villeneuve d'Ascq, France
}

\begin{abstract}
SUMMARY
Stabilization rates of power-integrator chains are easily regulated. It provides a framework for acceleration of uncertain multi-input multi-output (MIMO) dynamic systems of known relative degrees (RDs). The desired rate of the output stabilization (sliding-mode (SM) control) is ensured for an uncertain system, if its RD is known, and a rough approximation of the high-frequency gain matrix is available. The uniformly bounded convergence time (fixed-time stability) is obtained as a particular case. The control can be kept continuous everywhere accept the SM set, if the partial RDs are equal. Similarly uncertain smooth systems of complete MIMO RDs (i.e. lacking zero dynamics) are stabilized by continuous control at their equilibria in finite time and also accelerated. Output-feedback controllers are constructed. Computer simulation demonstrates the efficiency of the proposed approach.
\end{abstract}

Copyright (c) 0000 John Wiley \& Sons, Ltd.

Received ...

KEY WORDS: Sliding mode control; Homogeneous systems; Finite-time stability, Uncertain systems

\section{INTRODUCTION}

The output-regulation task is reduced to the output stabilization following a proper output redefinition. In its turn the output dynamics of a Single-Input Single-Output (SISO) system in general is described by an integrator chain, whereas the uncertainty, the overall system influence and the control are shifted to the last integrator [23]. The number of integrators is the relative degree (RD) [23], i.e. the least order of the output time derivative which explicitly contains the control with a non-zero coefficient. The RD can be considered as a design parameter [20,31], or is often known even under uncertainty conditions [12, 24, 48, 49].

In the Multi-Input Multi-Output (MIMO) case the numbers of inputs (controls) and outputs are assumed equal. The output dynamics are reduced to the corresponding number of integrator chains connected through the last integrators where the controls appear. Chain's lengths constitute the vector $\mathrm{RD}$, whereas the control coefficients constitute a non-singular matrix [23], called the high-frequency gain (HFG) matrix.

Consider finite-time (FT) output stabilization. If the uncertainty vanishes with the output, the task is reformulated as stabilization at an equilibrium. In the opposite case a discontinuous control is required, and sliding-mode (SM) control (SMC) is to be applied. In both cases the homogeneity theory is one of the main design approaches [3, 5, 27, 41], and the homogeneity degree is negative. In this paper we consider a general method of accelerating such stabilization

*Correspondence to: levant@post.tau.ac.il

Copyright (C) 0000 John Wiley \& Sons, Ltd.

Prepared using rncauth.cls [Version: 2010/03/27 v2.00] 
under uncertainty conditions, provided a homogeneous FT-stabilization feedback control is already available.

SMC keeps the outputs (sliding variables) at zero by means of high-frequency control switching. SMs are accurate and insensitive to disturbances, but also feature the so-called chattering effect due to high control-switching frequency [4, 7, 14, 19, 30, 47]. Standard SMC needs the partial RDs to be 1 . High order sliding modes (HOSMs) [4, 26, 9, 11, 18, 24, 27, 42, 39] are effective for any RD.

In the SISO case a universal output-feedback HOSM control [26, 27, 28] stabilizes the integrator chain in FT. Only the number of the integrators, i.e. the $\mathrm{RD}$, is actually required to be known. Additional integrators in the feedback artificially increase the RD and can be used to remove dangerous types of chattering $[4,30]$.

In the MIMO case the HFG matrix is usually to be available exactly or with high precision to use HOSM controls. In particular, the implicit-Lyapunov-function method [43, 44, 45] requires the exact knowledge of the HFG matrix. Contrary to this papers [9, 34] only assume a variable nominal value of the HFG matrix to be available and provide for the FT convergence to the SM. The MIMO SMC $[34,45]$ is homogeneous.

Whereas SMC corresponds to zero weights of the homogeneous feedback control, FT stabilization at equilibrium assumes positive control weights $[3,6,21,22$, 40]. Practical applications in particular include robotics and spacecraft attitude control [12, 49].

A standard continuous feedback $[29,32]$ stabilizes uncertain smooth systems at their equilibria in FT. No standard system form is required. The main assumption is that the system possesses a known permanent RD and lacks zero dynamics. Like in the MIMO SMC case, a nonsingular nominal HFG matrix is to be available.

FT-stable homogeneous systems are known to slowly converge at large distances. A SISO second-order SMC acceleration technique was proposed in [13], but it is extendable neither to general second-order SMC nor to the FT equilibrium stabilization. The acceleration-factor method for the homogeneous MIMO equilibrium stabilization was recently proposed in [32].

The FT and fixed-time convergence are provided by acceleration of asymptotically stable (not FT stable) homogeneous systems of non-negative degrees in the recent papers $[15,16]$, a similar idea was applied in [1]. The corresponding acceleration factor necessarily tends to infinity as the equilibrium is approached.

Acceleration of general FT-stable homogeneous output dynamics is considered in this paper. We start with a system consisting of a number of power-integrator chains coupled through the last equations and show that any homogeneous FT-stabilizing control is easily accelerated by a scalar factor. Then the time-variable acceleration is applied to MIMO HOSMs and uncertain smooth systems with FT-stable equilibria.

Any prescribed dependence of the transient time on the homogeneous norm of the stabilization error can be maintained along system trajectories. Fixed-time convergence is obtained as a particular case, when the convergence-time function is uniformly bounded.

The acceleration factor switching yields piece-wise-homogeneous dynamics, which allows output-feedback control versions. Contrary to $[15,16]$ at the last stage the acceleration factor is kept at 1 , which restores the original system convergence rate and diminishes the system stress. Simulation demonstrates the application of the method.

\section{WEIGHTED HOMOGENEITY NOTIONS}

Recall that a solution of a differential inclusion (DI) $\dot{x} \in F(x), F(x) \subset T_{x} \mathbb{R}^{n}$, is defined as any locally absolutely continuous function $x(t)$, satisfying the DI for almost all $t$. We call a DI $\dot{x} \in F(x)$ Filippov $D I$, if $F(x) \subset T_{x} \mathbb{R}^{n}$ is non-empty, compact and convex for any $x$, and $F$ is an upper-semicontinuous set function.

Here $T_{x} \mathbb{R}^{n}$ is the tangential space to $\mathbb{R}^{n}$ at $x$ [23]. For fixed coordinates it can be formally identified with $\mathbb{R}^{n}$ itself. The upper semicontinuity of $F(x)$ means that the maximal distance of 
the points of $F(x)$ from the set $F(y)$ tends to zero, as $x \rightarrow y$. The upper (lower) semi-continuity of a scalar function $\phi: \mathbb{R}^{n} \rightarrow \mathbb{R}$ means that $\limsup _{x \rightarrow y} \phi(x) \leq \phi(y)\left(\liminf _{x \rightarrow y} \phi(x) \geq \phi(y)\right)$.

It is well-known that Filippov DIs have most standard features except the uniqueness of solutions [17]. Asymptotically stable Filippov DIs have smooth Lyapunov functions [8].

Introduce the weights $\operatorname{deg} x_{i}=m_{i}>0$ of the coordinates $x_{1}, x_{2}, \ldots, x_{n}$ in $\mathbb{R}^{n}$. Define the dilation [3, 6] $d_{\kappa}:\left(x_{1}, x_{2}, \ldots, x_{n}\right) \mapsto\left(\kappa^{m_{1}} x_{1}, \kappa^{m_{2}} x_{2}, \ldots, \kappa^{m_{n}} x_{n}\right)$, where $\kappa>0$. Recall [3, 25] that a function $f: \mathbb{R}^{n} \rightarrow \mathbb{R}^{m}$ is said to have the homogeneity degree (weight) $q_{h} \in \mathbb{R}$, $\operatorname{deg} f=q_{h}$, if the identity $f(x)=\kappa^{-q_{h}} f\left(d_{\kappa} x\right)$ holds for any $x \in \mathbb{R}^{n}$ and $\kappa>0$.

A vector-set field $F(x) \subset T_{x} \mathbb{R}^{n}$ (DI $\dot{x} \in F(x)$ ) is called homogeneous of the degree $q_{h} \in \mathbb{R}$ [27], if the identity $F(x)=\kappa^{-q_{h}} d_{\kappa}^{-1} F\left(d_{\kappa} x\right)$ holds for any $x \in \mathbb{R}^{n}$ and $\kappa>0$.

The homogeneity of a vector-set field $F(x)$ can equivalently be defined as the invariance of the DI $\dot{x} \in F(x)$ with respect to the combined time-coordinate transformation $G_{\kappa}:(t, x) \mapsto$ $\left(\kappa^{p_{h}} t, d_{\kappa} x\right), \quad \kappa>0$, where $p_{h}, p_{h}=-q_{h}$, might naturally be considered as the weight of $t$. Indeed, the homogeneity condition can be rewritten as $\dot{x} \in F(x) \Leftrightarrow \frac{d\left(d_{\kappa} x\right)}{d\left(\kappa^{p} h t\right)} \in F\left(d_{\kappa} x\right)$.

The differential equation $\dot{x}=f(x) \in T_{x} \mathbb{R}^{n}, \dot{x}_{i}=f_{i}(x), i=1, \ldots, n$, can be considered as the DI $\dot{x} \in\{f(x)\}$. Then the above definition is reduced to the standard definition $\operatorname{deg} f_{i}=$ $\operatorname{deg} \dot{x}_{i}=\operatorname{deg} x_{i}-\operatorname{deg} t=m_{i}+q_{h}[3,25]$.

The non-zero homogeneity degree $q_{h}$ of a vector-set field can always be scaled to \pm 1 by an appropriate proportional change of the weights $m_{1}, \ldots, m_{n}, \operatorname{deg} x_{i}=m_{i}$. The upper semicontinuity of the DI inevitably imposes the condition $m_{i} \geq-q_{h}$ for all coordinates $x_{i}$.

The contractivity [27,33] of a homogeneous Filippov DI is equivalent to the existence of $T>0, R>r>0$, such that all solutions starting in the ball $\|x\| \leq R$ at the time 0 are in the smaller ball $\|x\| \leq r$ at the time $T$.

Let a Filippov DI be homogeneous of a negative homogeneity degree. Then FT stability and asymptotic stability features are equivalent $[27,35,41]$, and both are equivalent to the contractivity [27]. The maximal (minimal) stabilization time is a well-defined upper (lower) semi-continuous function of the initial conditions [35].

The FT stability of the homogeneous Filippov DI $\dot{x} \in F(x)$ implies the negativeness of the homogeneity system degree $q_{h}<0$, and respectively $\operatorname{deg} \dot{x}_{i}=m_{i}+q_{h} \geq 0, i=1, \ldots, n$ [35].

A homogeneous norm $\|x\|_{h}$ is by definition any positive-definite continuous homogeneous function of the weight 1 . It is not a real norm (the triangle inequality does not hold). The standard norm $\|\cdot\|$ used in this paper is the Euclidian one.

\section{ACCELERATION OF POWER INTEGRATORS}

In the following we use the convenient notation extending the standard power functions: $\forall s \neq 0\lfloor s\rceil^{\gamma}=|s|^{\gamma} \operatorname{sign} s ; \forall \gamma>0\lfloor 0\rceil^{\gamma}=0 ;\lfloor s\rceil^{0}=\operatorname{sign} x$.

One of the simplest homogeneous systems is a power integrator chain [38, 21]. Consider its MIMO variant

$$
\begin{aligned}
& \dot{z}_{i, 1}=\left\lfloor z_{i, 2}\right\rceil^{p_{i, 1}}, \\
& \ldots, \\
& \dot{z}_{i, r_{i}-1}=\left\lfloor z_{i, r_{i}}\right\rceil^{p_{i, r_{i}-1}}, \\
& \dot{z}_{i, r_{i}} \in F_{i}(z), i=1, \ldots, n_{z}, z=\left(z_{1}, \ldots, z_{n_{z}}\right)
\end{aligned}
$$

where $p_{i, j}>0$. Naturally $F_{i}(z) \subset \mathbb{R}$ is a closed segment, upper semicontinuous in $z \in$ $\mathbb{R}^{r_{1}+\ldots+r_{m}}$, so that (3.1) is a Filippov DI.

Choose -1 as the system homogeneity degree, and denote $\operatorname{deg} z_{i, j}=m_{i, j}$ and $\operatorname{deg} \dot{z}_{i, r_{i}}=$ $q_{i} \geq 0$. It implies the relations

$$
m_{i, r_{i}}=1+q_{i}, m_{i, j-1}=1+m_{i, j} p_{i, j-1}, j=r_{i}-1, \ldots, 1,
$$

which recursively define all weights, $m_{i, j}>1$ for all $j<r_{i}, m_{i, r}=1+q_{i} \geq 1, i=1, \ldots, n_{z}$, $j=1, \ldots, r_{i}$. 
Introduce the homogeneous norm

$$
\begin{aligned}
& \left\|z_{i}\right\|_{h}=\left(\left|z_{i, 1}\right|^{\frac{\rho_{z}}{m_{i, 1}}}+\ldots+\left|z_{i, r_{i}}\right|^{\frac{\rho_{z}}{m_{i, r_{i}}}}\right)^{\frac{1}{\rho_{z}}}, \rho_{z}>\max _{i, j} m_{i, j}, \\
& \|z\|_{h}=\left\|z_{1}\right\|_{h}+\ldots+\left\|z_{n_{z}}\right\|_{h} .
\end{aligned}
$$

That norm $\|z\|_{h}$ is continuously differentiable everywhere except $z=0$.

\subsection{Lipschitz property of homogeneous norms and extendability of solutions}

Consider the non-autonomous modification of (3.1)

$$
\begin{aligned}
& \dot{z}_{i, 1}=\left\lfloor z_{i, 2}\right\rceil^{p_{i, 1}}, \\
& \ldots, \\
& \dot{z}_{i, r_{i}-1}=\left\lfloor z_{i, r_{i}}\right\rceil^{p_{i, r_{i}-1}}, \\
& \dot{z}_{i, r_{i}} \in \alpha(t) F_{i}(z), i=1, \ldots, n_{z},
\end{aligned}
$$

where $\alpha(t)$ is some locally essentially-bounded Lebesgue-measurable function.

We formally add $\dot{t}=1$ and understand generalized solutions of (3.3) as the solutions of the enlarged Filippov DI obtained when $\alpha(t)$ in (3.4) is replaced with the upper-semicontinuous set function $A(t)[-1,1]$, where $A(t)=\operatorname{ess~limsup}_{s \rightarrow t}|\alpha(t)|$.

The following simple lemma is only true if the homogeneity degree -1 of (3.1) is chosen (respectively $\operatorname{deg} t=1$ ). DI (3.1) is allowed to be unstable.

Note that $\max _{z \in \Omega, w \in F_{i}(z)}|w|$ is well defined for any compact set $\Omega$ due to the upper semicontinuity and compactness of $F_{i}(z)$.

Lemma 1. Let system (3.1) be homogeneous with the homogeneity degree -1 , and let $\|z\|_{h}$ be any homogeneous norm continuously differentiable everywhere except the origin $z=0$. Then there exist such constants $L_{0}, L_{1}$ only depending on the chosen norm that

$$
\left|\frac{d}{d t}\right|\left|z(t) \|_{h}\right| \leq L_{0}+L_{1} M_{F} A(t), M_{F}=\max _{\|z\|_{h}=1, w \in F_{i}(z), i=1, \ldots, m}|w|
$$

holds for almost all t along any generalized solution of (3.3).

Proof

Due to the homogeneity of (3.1) get

$$
\operatorname{deg} \frac{\partial\|z\|_{h}}{\partial z_{i, j}} \dot{z}_{i, j}=1-\operatorname{deg} z_{i, j}+\operatorname{deg} z_{i, j}-1=0, \text { for } j=1, \ldots, r_{i}-1, \operatorname{deg} \frac{\partial\|z\|_{h}}{\partial z_{i, r_{i}}}=-q_{i} .
$$

On the other hand, due to the homogeneity of (3.1) $\max _{i, w \in F_{i}(z)}\|w\|=M_{F}\|z\|_{h}^{q_{i}}$. Thus

$$
\left.\left|\frac{d}{d t}\right||z(t)|\right|_{h}\left|\leq \sum_{i \leq n_{z}, j<r_{i}}\right| \frac{\partial\|z\|_{h}}{\partial z_{i, j}}|| \dot{z}_{i, j}\left|+A(t) M_{F}\|z\|_{h}^{q_{i}} \sum_{i \leq n_{z}}\right| \frac{\partial\|z\|_{h}}{\partial z_{i, r_{i}}} \mid,
$$

where the first sum and the coefficient of $A(t)$ are homogeneous functions of degree 0 , which are continuous for $z \neq 0$, and, therefore, are bounded.

Since any negative homogeneity degree is shifted to -1 by an appropriate proportional change of the coordinate weights, Lemma 1 implies indefinite extendability in time [2] for any generalized solution of (3.3), if the homogeneity degree of system (3.1) is negative.

\subsection{Convergence acceleration}

Now consider the same system (3.1) in the new time $t_{1}=t / \mu$, where $\mu \geq 1$. We get

$$
\begin{aligned}
& \dot{z}_{i, 1}=\mu\left\lfloor z_{i, 2}\right\rceil^{p_{i, 1}}, \\
& \ldots, \\
& \dot{z}_{i, r_{i}-1}=\mu\left\lfloor z_{i, r_{i}}\right\rceil^{p_{i, r_{i}-1},} \\
& \dot{z}_{i, r_{i}} \in \mu F_{i}(z) .
\end{aligned}
$$


Introducing the notation $\tilde{z}_{i, 1}=z_{i, 1}, \tilde{z}_{i, 2}=\mu^{\frac{1}{p_{i, 1}}} z_{i, 2}, \ldots$, obtain the coordinate transformation $\tilde{z}_{i, j}=\mu^{\nu_{i, j}} z_{i, j}$, for some $\nu_{i, j} \geq 0, i=1, \ldots, n_{z}, j=1, \ldots, r_{i}$. In the new coordinates system (3.5) turns to be

$$
\begin{aligned}
& \dot{\tilde{z}}_{i, 1}=\left\lfloor\tilde{z}_{i, 2}\right\rceil^{p_{i, 1}}, \\
& \ldots, \\
& \ddot{\tilde{z}}_{i, r_{i}-1}=\left\lfloor\tilde{z}_{i, r_{i}}\right\rceil^{p_{i, r_{i}-1}}, \\
& \dot{\tilde{z}}_{i, r_{i}} \in \mu^{1+\nu_{i, r_{i}}} F_{i}\left(\ldots, \mu^{-\nu_{i, j}} \tilde{z}_{i, j}, \ldots\right)=\tilde{F}(\tilde{z}) .
\end{aligned}
$$

Recall that for any FT stable homogeneous system stabilization time from any point on the homogeneous sphere $\|z\|_{h}=1$ belongs to some segment $\left[T_{*}, T *\right]$, where the stabilization times $T_{*}, T^{*}$ are indeed obtained on some solutions [35]. The following simple lemma plays important role in this article.

Lemma 2. Fix homogeneous norm (3.2). Let system (3.1) be FT stable and homogeneous with the homogeneity degree -1 , and the stabilization times from the homogeneous sphere $\|z\|_{h}=1$ belong to $\left[T_{*}, T^{*}\right]$. Then for any $\mu \geq 1$ solutions of (3.6) starting from the homogeneous sphere $\|\tilde{z}\|_{h}=R$ have stabilization times belonging to $\left[\left(\min _{i, j} \mu^{-\nu_{i, j} / m_{i, j}}\right) T_{*}, T^{*}\right] R / \mu$.

Proof

Obviously, $z_{i, j}=\mu^{-\nu_{i, j}} \tilde{z}_{i, j}$, and

$$
\left(\min _{i, j} \mu^{-\frac{\nu_{i, j}}{m_{i, j}}}\right)|| \tilde{z}\left\|_{h} \leq\right\| z \|_{h}=\left[\Sigma_{i, j} \mu^{-\frac{\rho_{z} \nu_{i, j}}{m_{i, j}}}\left|\tilde{z}_{i, j}\right|^{\frac{\rho_{z}}{m_{i, j}}} \frac{1}{\rho_{z}} \leq\|\tilde{z}\|_{h} .\right.
$$

Thus, (3.2) and $\|\tilde{z}\|_{h}=R$ imply $\|z\|_{h} \in\left[\min _{i, j} \mu^{-\nu_{i, j} / m_{i, j}}, 1\right] R$. Dynamics of $z$ obeys (3.5). Respectively the transient times are $\mu$ times less than those of (3.1). The rest of the proof follows from the remark that since the minimal/maximal stabilization-time function is homogeneous of the weight $1[27,35]$, then for any $\tilde{R}>0$ the stabilization times of (3.1) from the sphere $\|z\|_{h}=\tilde{R}$ belong to $\left[T_{*}, T^{*}\right] \tilde{R}$.

Thus, one can easily accelerate any MIMO power integrator chain (3.1), in particular, chains closed by the controllers by Hong [21] or Bhat, Bernstein [6]. Note that the normal controllability forms of linear systems belong to the class (3.1) if the control feedback is considered as a part of $F_{i}$. Then pure integrators with $p_{i, j}=1$ are achieved. Pure integrators also naturally appear when systems with well defined relative degrees are considered [23].

Consider the important case $p_{i, j}=1$, then $\nu_{i, j}=j-1, m_{i, j}=r_{i}-j+1+q_{i}$. Respectively then Lemma 2 states that the stabilization times belong to $\left[\mu^{-\max \frac{r_{i}+q_{i}-1}{1+q_{i}}} T_{*}, T^{*}\right] R / \mu$, and if also $\forall i q_{i}=0$ then they belong to $\left[\mu^{1-\max r_{i}} T_{*}, T^{*}\right] R / \mu$.

The acceleration methods are further developed for uncertain disturbed pure-integrator chains, but they are exactly in the same way applicable to the general power integrators (3.1).

\section{PRELIMINARIES: HOMOGENEOUS SMC}

This section shortly introduces the basics of homogeneous SMs $[9,26,27,34]$. Though technically not new, the following concentrated presentation cannot be found in literature.

\subsection{The SMC problem statement}

The following "black box control" problem formulation is traditional in the SISO case $[4,11,18,26,27,39,42]$ and treats the relative degree as a key parameter for both the model and the SM control design $[20,24,31,35]$. Its natural generalization to the MIMO systems $[9,34,45]$ seemingly becomes standard as well.

Consider a MIMO dynamic system of the form

$$
\dot{x}=a(t, x)+b(t, x) u, \sigma=\sigma(t, x),
$$


where $x \in \mathbb{R}^{n}, u \in \mathbb{R}^{m}$ is the control, $m \leq n, a, b$ and $\sigma$ are some uncertain smooth functions. The output $\sigma(t, x(t)) \in \mathbb{R}^{m}$ is available in real time for $t \geq t_{0}$. The system is understood in the Filippov sense [17] in order to allow discontinuous controls.

The task is to establish and keep $\sigma \equiv 0$. Further we solve this problem under the additional requirement to conform an upper estimation of the convergence time given in advance as a function of initial conditions.

The uncertain system (4.1) features the known integer vector $r \in \mathbb{N}^{m}$, real numbers $p_{0}, C$, $K_{m}, K_{M}$ and a nonsingular $m \times m$ matrix $G(t, x)$ available in real time.

Denote $\sigma=\left(\sigma_{1}, \ldots, \sigma_{m}\right), \sigma^{(r)}=\left(\sigma_{1}^{\left(r_{1}\right)}, \ldots, \sigma_{m}^{\left(r_{m}\right)}\right)^{T}, \vec{\sigma}_{i}=\left(\sigma_{i}, \dot{\sigma}_{i}, \ldots, \sigma_{i}^{\left(r_{i}-1\right)}\right), \vec{\sigma}=\left(\vec{\sigma}_{1}, \ldots, \vec{\sigma}_{m}\right)$.

The vector $r=\left(r_{i}, \ldots, r_{m}\right)$ is the relative degree [23] of the system (4.1), and is assumed to be constant and known. That means that

$$
\sigma^{(r)}=h(t, x)+g(t, x) u,
$$

where $h(t, x), g(t, x)$ are some smooth functions, which can be expressed via Lie derivatives. The function $g(t, x)$ is a nonsingular $m \times m$ matrix [23], $g_{i j}=\frac{\partial}{\partial u_{j}} \sigma_{i}^{\left(r_{i}\right)}$. Obviously, $\sigma_{i}^{(j)}(t, x)$, $j=0,1, \ldots, r_{i}-1$, are smooth functions. Solutions of the system are assumed indefinitely extendable in time provided $g(t, x(t)) u(t)$ remains bounded along the trajectory.

Suppose that whereas the exact dynamics (4.1) and (4.2) are unknown, the representation

$$
g(t, x)=K(t, x)(G(t, x)+\Delta g(t, x))
$$

is available, where $K$ is some uncertain positive scalar factor, $G(t, x)$ approximately defines the "direction" of $g, \operatorname{det} G \neq 0$, and $\Delta g$ is some uncertain "directional" disturbance. The matrix $G$ can be any Lebesgue-measurable function of some observable coordinates.

It is assumed that

$$
\left\|\triangle g(x, t) G^{-1}(x, t)\right\|_{1} \leq p_{0}<1,
$$

where the norm $\|A\|_{1}, A=\left(a_{i j}\right)$, is defined as $\|A\|_{1}=\max _{i} \sum_{j}\left|a_{i j}\right|$.

The term $h$ and the factor $K$ are supposed to satisfy the inequalities

$$
\|h(t, x)\| \leq C, K_{m} \leq K(t, x) \leq K_{M} .
$$

We stabilize $\sigma$ at zero by means of a feedback control of the form $u=U(t, x)$. It follows from (4.2) that no continuous feedback of such a form is capable of keeping $\sigma \equiv 0$ for all possible $h$ if $C>0$.

Recall [26] that if the $r$-sliding set ( $r$ th-order sliding set) $\vec{\sigma}=0$ is a non-empty integral set, then the motion on the set is said to be in the $r$ th-order SM $\left(r\right.$-SM). The vector $r=\left(r_{i}, \ldots, r_{m}\right)$ is called the sliding order [26].

Thus in order to solve the stated problem one needs to establish and keep the $r$-SM $\sigma=0$. The control $u=U(t, x)$ is called quasi-continuous [28,37] if the function $U$ is continuous whenever $\vec{\sigma} \neq 0$. Such controllers feature less chattering in practical applications, since due to the inevitable measurement noises and delays the discontinuity condition $\vec{\sigma}=0$ is never fulfilled if $m>1$ or $\max r_{i}>1$.

\subsection{Sliding mode homogeneity}

The input-output dynamics (4.2) are still too complicated. The next standard step is to simplify them $[9,34]$. Let

$$
\begin{aligned}
& u=G^{-1}(t, x) v, v=\left(v_{1}, \ldots, v_{m}\right)^{T}, \\
& v_{i}=\alpha V_{i}(\vec{\sigma}), \alpha>0,\left|V_{i}(\vec{\sigma})\right| \leq 1, i=1,2, \ldots, m,
\end{aligned}
$$

where $v$ is a virtual control, whose components $v_{i}=\alpha V_{i}(\vec{\sigma})$ have the same magnitude $\alpha$. Then due to (4.2)-(4.5) any solution of (4.1) satisfies the inclusion

$$
\sigma_{i}^{\left(r_{i}\right)} \in[-C, C]+\alpha\left[K_{m}, K_{M}\right]\left(\left[-p_{0}, p_{0}\right]+V_{i}(\vec{\sigma})\right), i=1, \ldots, m .
$$


Solutions of (4.8) are understood as the solutions of the DI obtained after the substitution of the upper-semi-continuous segment function $\left.V_{F i}(\vec{\sigma})\right)$ for $V_{i}(\vec{\sigma})$. Here $V_{F i}$ is obtained by the Filippov procedure [17].

The main control design idea is to choose the control (4.7) so that the closed-loop differential inclusion (4.7), (4.8) becomes homogeneous.

Due to the presence of the segment $[-C, C]$ in (4.8) the homogeneity weight of the right hand side can only be 0 . Thus, $\operatorname{deg} \sigma_{i}^{\left(r_{i}\right)}=\operatorname{deg} \sigma_{i}^{\left(r_{i}-1\right)}-\operatorname{deg} t=0$. Taking the homogeneity degree -1 (i.e. $\operatorname{deg} t=1$ ), obtain that $\operatorname{deg} \sigma_{i}^{\left(r_{i}-1\right)}=1$. Thus $\operatorname{deg} \sigma_{i}^{\left(r_{i}-2\right)}=\operatorname{deg} \sigma_{i}^{\left(r_{i}-1\right)}+1=2$, etc. Respectively $\operatorname{deg} t=1, \operatorname{deg} \sigma_{i}^{(j)}=r_{i}-j, i=1, \ldots, m, j=0, \ldots, r_{i}-1$. That homogeneity, or any other one with proportional weights is called $r$-sliding homogeneity [27].

The corresponding dilations are

$$
\begin{aligned}
& d_{\kappa}: \vec{\sigma} \mapsto\left(d_{1, \kappa} \vec{\sigma}_{1}, \ldots, d_{m, \kappa} \vec{\sigma}_{m}\right), \\
& d_{i, \kappa}: \vec{\sigma}_{i} \mapsto\left(\kappa^{r_{i}} \sigma_{i}, \kappa^{r_{i}-1} \dot{\sigma}_{i}, \ldots, \kappa \sigma_{i}^{\left(r_{i}-1\right)}\right) .
\end{aligned}
$$

Since $\operatorname{deg} \sigma_{i}^{\left(r_{i}\right)}=0$, also $\operatorname{deg} V_{i}=0$. Respectively, control (4.7) is called $\left(r_{1}, \ldots, r_{m}\right)$-sliding homogeneous, if $V\left(d_{\kappa} \vec{\sigma}\right) \equiv V(\vec{\sigma})$. The resulting SM, if exists, is also called homogeneous.

The corresponding homogeneous norms can be chosen as follows

$$
\begin{aligned}
\left\|\vec{\sigma}_{i}\right\|_{h} & =\left(\left|\sigma_{i}\right|^{\frac{\bar{\rho}_{i}}{r_{i}}}+\left|\dot{\sigma}_{i}\right|^{\frac{\bar{\rho}_{i}}{r_{i}-1}}+\ldots+\left|\sigma_{i}^{\left(r_{i}-1\right)}\right|^{\bar{\rho}_{i}}\right)^{\frac{1}{\bar{\rho}_{i}}}, i=1, \ldots, m, \\
\|\vec{\sigma}\|_{h} & =\left\|\vec{\sigma}_{1}\right\|_{h}+\left\|\vec{\sigma}_{2}\right\|_{h}+\ldots+\left\|\vec{\sigma}_{m}\right\|_{h}, \bar{\rho}_{i}>0 .
\end{aligned}
$$

Note that if $\bar{\rho}_{i}>r_{i}$ the norm $\left\|\vec{\sigma}_{i}\right\|_{h}$ is differentiable everywhere except $\vec{\sigma}_{i}=0$.

\subsection{Single-input single-output (SISO) homogeneous SMs.}

In the SISO case $m=1, \sigma, u \in \mathbb{R}, r \in \mathbb{N}, g=G= \pm 1, \Delta g=0$. Naturally $g=1, u=v$ are taken $[26,27]$, and (4.6)-(4.8) become

$$
\sigma^{(r)} \in[-C, C]+\left[K_{m}, K_{M}\right] u .
$$

There are a lot of controllers of the form

$$
u=-\alpha \Psi_{r}(\vec{\sigma}),\left|\Psi_{r}(\vec{\sigma})\right| \leq 1, \alpha>0,
$$

stabilizing (4.11) in FT for sufficiently large $\alpha$. Hence they solve the problem for any natural number $r$ and $C, K_{m}, K_{M}[11,27,28,37,44]$. Here and further the derivatives of $\sigma$ can be provided by robust exact differentiators (Section 4.5).

The following homogeneous "relay polynomial" controllers [10] are probably the most simple controllers of such kind:

$$
u=-\alpha \operatorname{sign}\left(\left\lfloor\sigma^{(r-1)}\right\rceil^{\frac{\rho_{0}}{1}}+\beta_{r-2}\left\lfloor\left.\sigma^{(r-1)}\right|^{\frac{\rho_{0}}{2}}+\ldots+\beta_{0}\lfloor\sigma\rceil^{\frac{\rho_{0}}{r}}\right) .\right.
$$

The quasi-continuous version of the above polynomial controller is

$$
u=-\alpha \frac{\left\lfloor\sigma^{(r-1)}\right\rceil^{\frac{\rho_{0}}{1}}+\beta_{r-2}\left\lfloor\sigma^{(r-1)}\right\rceil^{\frac{\rho_{0}}{2}}+\ldots+\beta_{0}\lfloor\sigma\rceil^{\frac{\rho_{0}}{r}}}{\left|\sigma^{(r-1)}\right|^{\frac{\rho_{0}}{1}}+\beta_{r-2}\left|\sigma^{(r-1)}\right|^{\frac{\rho_{0}}{2}}+\ldots+\beta_{0}|\sigma|^{\frac{\rho_{0}}{r}}} .
$$

Any $\rho_{0}>0$ can be taken, $\beta_{j}>0$. The corresponding parametric sets $\left\{\beta_{0}, \ldots, \beta_{r-2}\right\}$ are arbitrary for $r=1,2$. For $r=3,4,5$ and $\rho_{0}=r$ one can choose the following valid sets: 3$)\{1,1\} ; 4$ ) $\{1,2,2\} ; 5)\{1,3,5,6\}$.

Note that the idea of the quasi-continuous SMC (4.12) is to keep the trajectories of (4.11), (4.12) close to the set $\Psi_{r}(\vec{\sigma})=0$, corresponding to a FT-stable $r$-sliding homogeneous differential equation on $\sigma$ of the order $r-1$ [37]. 


\subsection{Multi-input multi-output (MIMO) homogeneous SMs.}

Return to the case $\sigma \in \mathbb{R}^{m}, r \in \mathbb{N}^{m}, \vec{\sigma}=\left(\vec{\sigma}_{1}^{T}, \ldots, \vec{\sigma}_{m}^{T}\right)^{T}$. Close the inclusion (4.8) with partial controls of the form

$$
V_{i}(\vec{\sigma})=-\tilde{\Psi}_{i, r_{i}}\left(\vec{\sigma}_{i}\right),\left|\tilde{\Psi}_{i, r_{i}}\right| \leq 1, \operatorname{deg} \tilde{\Psi}_{i, r_{i}}=0,
$$

where $\tilde{\Psi}_{i, r_{i}}$ appears in a stabilizing SISO control of the form (4.12). As a result, obtain a completely decoupled closed-loop $r$-sliding homogeneous inclusion. Any SISO $r_{i}$-SM controller of the form (4.12) can be utilized. In the case the right hand side $\tilde{\Psi}_{i, r_{i}}$ is taken from (4.13), due to condition (4.4) it will be FT stable for sufficiently large $\alpha$. Indeed, in that case $V_{i}= \pm 1$, and it dominates in (4.8), since $p_{0}<1$.

One would like to build MIMO quasi-continuous controllers. Naturally it only makes sense if the function $G(t, x)$ is continuous. For that end one can apply controllers like (4.14). In particular,

$$
V_{i}(\vec{\sigma})=-\operatorname{sat}\left(\eta_{i} \tilde{\Psi}_{i, r_{i}}\left(\vec{\sigma}_{i}\right)\right), \eta_{i} \geq 1, \operatorname{sat} \omega=\max (-1, \min (1, \omega)),
$$

works with (4.8) for some large enough $\eta$ [34]. The idea is that the saturated function $\operatorname{sat}\left(\eta_{i} \Psi_{i, r_{i}}\right)$ takes on values close to \pm 1 in sufficiently small vicinity of $\Psi_{i, r_{i}}\left(\vec{\sigma}_{i}\right)=0$. Respectively $\vec{\sigma}_{i}$ is kept in a small vicinity of $\Psi_{i, r_{i}}\left(\vec{\sigma}_{i}\right)=0$, which provides for the FT convergence $\vec{\sigma}_{i} \rightarrow 0$. Unfortunately, in that case the resulting MIMO control still will not be quasi-continuous, since each control $v_{i}$ becomes discontinuous as the partial SM $\sigma_{i} \equiv 0$ is established, and it does not happen simultaneously for all $i$.

Note that controllers like (4.13) which only take values \pm 1 , are included in (4.16) as the particular case. In that case $\eta$ does not influence the control, and $\eta_{i}=1$ or any other value can be taken.

Quasi-continuous MIMO SMC. Let once more $\tilde{\Psi}_{i, r_{i}}$ be quasi-continuous SISO controllers, like in (4.12), $G$ be continuous. Then a MIMO quasi-continuous control is obtained by the combination of (4.6), (4.7) and

$$
\begin{aligned}
& V_{i}(\vec{\sigma})=-\Theta_{\theta}\left(\xi_{i}\right) \operatorname{sat}\left(\eta_{i} \tilde{\Psi}_{i, r_{i}}\left(\vec{\sigma}_{i}\right)\right), \eta_{i} \geq 1,0 \leq \theta \leq 1, \xi_{i}=\left\|\vec{\sigma}_{i}\right\|_{h} /\|\vec{\sigma}\|_{h}, \\
& \Theta_{\theta}(\omega)=\max (0, \min (1,2 \omega / \theta-1)) \text { for } \theta \neq 0, \Theta_{0}(\omega) \equiv 1 .
\end{aligned}
$$

Obviously $\operatorname{deg} \Theta_{\theta}\left(\xi_{i}(\vec{\sigma})\right)=0$. The control is not decoupled anymore. The function $\Theta_{\theta}\left(\xi_{i}\right)$ is continuous, and it equals 0 when $\left|\xi_{i}\right| \leq \theta / 2$. This prevents establishment of the partial SM $\sigma_{i}=0$ before the whole $r$-SM $\sigma=0$ is established. It is proved [32] that for sufficiently small $\theta$ the corresponding MIMO controller provides for the FT stability of (4.17), (4.8).

\subsection{Output-feedback control}

All the above controllers can be equipped with differentiators [26] yielding homogeneous output-feedback control.

Let the input signal have the form $\phi(t)=\phi_{0}(t)+\nu(t)$, where $\nu(t)$ is a bounded Lebesguemeasurable noise with unknown features, and $\phi_{0}(t)$ is an unknown base signal, almost everywhere satisfying $\left|\phi_{0}^{\left(k_{d}+1\right)}(t)\right| \leq L$, where $L$ is a known Lipschitz constant of $\phi_{0}^{\left(k_{d}\right)}$. The differentiator [26], presented in its recursive form as

$$
\begin{aligned}
& \dot{z}_{0}=-\lambda_{k_{d}} L^{\frac{1}{k_{d}+1}}\left\lfloor z_{0}-\phi(t)\right]^{\frac{k_{d}}{k_{d}+1}}+z_{1}, \\
& \dot{z}_{1}=-\lambda_{k_{d}-1} L^{\frac{1}{k_{d}}}\left\lfloor z_{1}-\dot{z}_{0}\right\rceil^{\frac{k_{d}-1}{k_{d}}}+z_{2}, \\
& \cdots \\
& \dot{z}_{k_{d}-1}=-\lambda_{1} L^{\frac{1}{2}}\left\lfloor z_{k_{d}-1}-\dot{z}_{k_{d}-2}\right\rceil^{\frac{1}{2}}+z_{k_{d}}, \\
& \dot{z}_{k_{d}}=-\lambda_{0} L \operatorname{sign}\left(z_{k_{d}}-\dot{z}_{k_{d}-1}\right),
\end{aligned}
$$

in FT provides for the estimations $z_{j}$ of the derivatives $\phi_{0}^{(j)}, j=0, \ldots, k_{d}$, which are exact if $\nu \equiv 0$. 
There are infinite sequences of parameters $\lambda_{j}$ each one yielding differentiators of all orders $k_{d} \geq 0[26]$. In particular, the sequence segment $\lambda_{0}=1.1, \lambda_{1}=1.5, \lambda_{2}=3, \lambda_{3}=5, \lambda_{4}=8$, $\lambda_{5}=12$ is sufficient for $k_{d} \leq 5$ [28]. Assuming that the sequence $\lambda_{j}$ is the same all over the paper, denote (4.18) by the equality $\dot{z}=D_{k_{d}}(z, \phi, L)$.

The differentiator error dynamics is homogeneous with the homogeneity degree -1 and $\operatorname{deg}\left(z_{j}-\phi^{(j)}\right)=k_{d}+1-j \quad[26]$. The accuracy $\left|z_{j}-\phi_{0}^{(j)}\right| \leq \mu_{j} L \delta^{k_{d}+1-j}, \quad \delta=$ $\max \left(\delta_{t},\left(\delta_{0} / L\right)^{1 /\left(k_{d}+1\right)}\right), \mu_{j} \geq 1$, is obtained for sampling time periods not exceeding $\delta_{t}>0$ and the maximal possible sampling error $\delta_{0} \geq 0$. This accuracy is asymptotically optimal in the presence of noises [26].

Since $G$ is measured, an output-feedback control is obtained from (4.6), (4.7) after replacing (4.7) with

$$
v_{i}=\alpha V_{i}(z), \dot{z}_{i}=D_{r_{i}-1}\left(z_{i}, \sigma_{i}, L\right), L \geq C+\alpha K_{M}\left(1+p_{0}\right) .
$$

Note that provided the control (4.7) is $r$-sliding homogeneous, i.e. $\operatorname{deg} V(\vec{\sigma})=0$, also the output feedback is homogeneous, and (4.8), (4.19) is homogeneous with the homogeneity degree -1 and $\operatorname{deg} \sigma_{i}^{(j)}=\operatorname{deg} z_{i j}=r_{i}-j, i=1, \ldots, m, j=0, \ldots, r_{i}-1$. Also the FT stability feature is preserved.

Let the sampling time periods and the sampling errors not exceed $\delta_{t}>0$ and $\delta_{0, i} \geq 0$ respectively, $\delta=\max \left(\delta_{t}, \max _{i} \delta_{0, i}^{1 / r_{i}}\right)$. Then, due to the homogeneity of the closed-loop MIMO system (4.8), (4.19), the resulting accuracy $\left|\sigma_{i}^{(j)}\right| \leq \omega_{i, j} \delta^{r_{i}-j}, \omega_{i, j}>0$, is established in FT $[27,35]$. It is asymptotically equivalent to $\|\vec{\sigma}\|_{h} \leq \omega \delta$ for some $\omega>0$.

\section{ACCELERATION OF MIMO SMC}

One of the main problems of the FT stabilization based on homogeneous constructions is the relatively slow convergence rate of trajectories at large distances from the origin.

Consider a MIMO system (4.1) satisfying the conditions (4.2)-(4.5). Let it be closed by homogeneous control (4.6), (4.7) or its output-feedback counterpart (4.19). Let the resulting input-output dynamics satisfy the homogeneous FTS differential inclusion (4.7), (4.8) or (4.19), (4.8).

Our goal is to modify the control (4.7) or (4.19) so as to ensure convergence of the trajectories of (4.8) to the origin $(r-\mathrm{SM}) \vec{\sigma}=0$ with some prescribed convergence rate. The convergence rate is to gradually slow down as $\vec{\sigma}(t)$ approaches the origin in order to diminish the system stress. In that aspect the minimal convergence rate is considered to be one provided by the original control (4.6), (4.7).

\subsection{Decoupled acceleration of MIMO SMC}

Consider the MIMO FT stabilizing control (4.6), (4.7), (4.16). Let

$$
\begin{aligned}
& \bar{r}=\max _{i} r_{i}, \underline{r}=\min _{i} r_{i}, \mu \geq 1, \\
& \left.v_{i}=\alpha \mu^{\bar{r}} V_{i}\left(\vec{\sigma}_{i} \mu^{-\bar{r}}\right)\right)=-\alpha \mu^{\bar{r}} \operatorname{sat}\left(\eta \tilde{\Psi}_{i, r_{i}}\left(\vec{\sigma}_{i} \mu^{-\bar{r}}\right)\right) .
\end{aligned}
$$

Thus, the trajectories of the closed loop system now satisfy the completely decoupled differential inclusion

$$
\sigma_{i}^{\left(r_{i}\right)} \in[-C, C]+\alpha \mu^{\bar{r}}\left[K_{m}, K_{M}\right]\left(\left[-p_{0}, p_{0}\right]-\operatorname{sat}\left(\eta \tilde{\Psi}_{i, r_{i}}\left(\vec{\sigma}_{i} \mu^{-\bar{r}}\right)\right)\right), i=1, \ldots, m .
$$

When $\mu=1$ it coincides with the FT stable inclusion (4.8), (4.16).

Let the stabilization time of any trajectory of (5.3) with $\mu=1$, starting within the homogeneous disk $\|\vec{\sigma}\|_{h} \leq 1$ belong to $\left[T_{*}, T^{*}\right], 0<T_{*} \leq T^{*}$. Note that $T_{*}, T^{*}$ exist due to [27]. The following lemma is the basis for the applied acceleration procedure. 
Lemma 3. Let $\mu \geq 1$, then control (4.7), (5.1), (5.2) provides for the convergence of any trajectory of system (4.8), (5.2) starting in the region $\|\vec{\sigma}\|_{h} \leq 1$ to the $r$-sliding mode $\vec{\sigma}=0$ in some time belonging to $\left[T_{*} / \mu^{\frac{\bar{r}\left(\frac{r}{r}-1\right)}{\underline{r}}}, T^{*}\right] / \mu$.

Proof

Consider the dynamics of $\sigma_{i}$. Since $\mu^{\bar{r}} \geq 1$, (5.3) implies partial FT-stable homogeneous DIs

$$
\sigma_{i}^{\left(r_{i}\right)} \in \alpha \mu^{\bar{r}}\left([-C, C]+\left[K_{m}, K_{M}\right]\left(\left[-p_{0}, p_{0}\right]-\operatorname{sat}\left(\eta \tilde{\Psi}_{i, r_{i}}\left(\vec{\sigma}_{i} \mu^{-\bar{r}}\right)\right)\right)\right) .
$$

Due to the $r_{i}$-sliding homogeneity property of $\tilde{\Psi}_{i, r_{i}}$, the following identity holds:

$$
\tilde{\Psi}_{i, r_{i}}\left(\vec{\sigma}_{i} \mu^{-\bar{r}}\right)=\tilde{\Psi}_{i, r_{i}}\left(\sigma_{i}, \dot{\sigma}_{i} \mu^{-\frac{\bar{T}}{r_{i}}}, \ldots, \sigma_{i}^{\left(r_{i}-1\right)} \mu^{-\left(r_{i}-1\right) \frac{\bar{r}}{r_{i}}}\right) .
$$

The lemma now follows from Lemma 2 applied separately for each $i$.

Obviously the convergence rate of each output $\sigma_{i}$ depends on its partial relative degree $r_{i}$. In particular, the fastest convergence is obtained for the output $\sigma_{i}$ with the largest value of $\bar{r} / r_{i}=\bar{r} / \underline{r}$.

Now introduce a convergence-rate function $T(R), R \geq 0, T(0)=0$. It is to be a monotonously growing, continuous and positive-definite function. Also let $R_{k}$ be a monotonously growing sequence, $R_{k} \rightarrow \infty, R_{0}=0, k=0,1,2, \ldots$. The goal is to ensure that all trajectories, at some time $t$ starting in or passing through the region $\|\vec{\sigma}\|_{h} \in\left[R_{k-1}, R_{k}\right], k \geq 1$, enter the $r$-sliding mode at the time not exceeding $t+T\left(R_{k}\right)$ (Fig. 5.1). Note that the fixed-time convergence $[43,44]$ is obtained in the particular case $\lim _{s \rightarrow \infty} T(s)=T_{M}<\infty$.

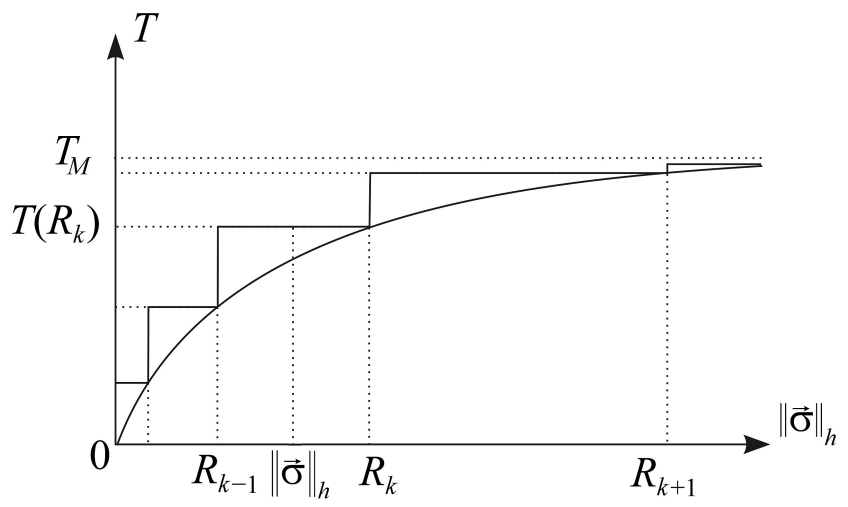

Figure 5.1: The convergence-rate function $T$, and its piece-wise-constant approximation. The case of the fixed-time convergence, $\lim _{R \rightarrow \infty} T(R)=T_{M}<\infty$.

For $k=0,1, \ldots$ define the functions

$$
\begin{aligned}
& n_{R}(\vec{\sigma})=k \Leftrightarrow\|\vec{\sigma}\|_{h} \in\left[R_{k-1}, R_{k}\right), R_{0}=0, R_{k}<R_{k+1}, \\
& \mu_{M}(\vec{\sigma})=\frac{\gamma R_{n_{R}(\vec{\sigma})}}{T\left(R_{n_{R}(\vec{\sigma})}\right)-T\left(R_{n_{R}(\vec{\sigma})-1}\right)}, \gamma>0 .
\end{aligned}
$$

Introduce the variable $\mu(t)$ that is left-hand continuous and features the discrete dynamics

$$
\begin{aligned}
\mu(t+0) & =\max \left\{1, \min \left[\mu(t), \mu_{M}(\vec{\sigma})\right]\right\}, \\
\mu\left(t_{0}\right) & =\max \left\{1, \mu_{M}(\vec{\sigma})\right\} .
\end{aligned}
$$

Obviously $\mu(t) \geq 1, \mu(t)$ is piece-wise constant and monotonously decreases. 
Theorem 1. Let $T_{\text {conv }}(t)$ be the convergence time to the $S M \sigma \equiv 0$ from the trajectory point $(t, x(t)), t \geq t_{0}$, of the system (4.1) under the control (4.6), (4.7), (4.16), (5.5), (5.6), (5.7), $\gamma \geq T^{*}$. Then along the trajectory it satisfies the inequality

$$
T_{\text {conv }}(t) \leq \frac{T^{*}}{\gamma} T\left(R_{n_{R}(\vec{\sigma}(t, x(t)))}\right) .
$$

The fixed-time convergence is obtained if $T(R)$ is uniformly bounded. While any $\gamma>0$ is applicable, it is reasonable to take $\gamma \geq T^{*}$, if $T^{*}$ can be estimated.

Proof

Projections of the considered trajectories to the space $\vec{\sigma}$ satisfy (4.8). From the first moment $t=t_{k}$ as $\vec{\sigma}(t)$ enters the ring set $\|\vec{\sigma}\|_{h} \in\left[R_{k-1}, R_{k}\right]$ the acceleration factor takes on the value

$$
\mu=\frac{\gamma R_{k}}{T\left(R_{k}\right)-T\left(R_{k-1}\right)},
$$

and it is kept constant until the trajectory of (4.8) enters $\|\vec{\sigma}\|_{h} \leq R_{k-1}$. According to Lemma 3 this value of $\mu$ ensures the convergence to zero of any trajectory starting in the ball $\|\vec{\sigma}\|_{h} \leq R_{k}$ in some time $\Delta t_{k} \leq\left(T\left(R_{k}\right)-T\left(R_{k-1}\right)\right) T^{*} / \gamma$. Due to (5.7) the relation is true also in the case $\mu_{M}<1$. On the way $\vec{\sigma}(t)$ enters the inner ball $\|\vec{\sigma}\|_{h} \leq R_{k-1}$, and the value of $\mu$ reduces, etc. It is important that though the trajectory $\vec{\sigma}(t)$ can leave the ball $\|\vec{\sigma}\|_{h} \leq R_{k}$ before entering the next inner ball $\|\vec{\sigma}\|_{h} \leq R_{k-1}$, the value of $\mu$ will not change, since it never grows according to (5.7). The resulting convergence time from the moment $t_{k}$ satisfies $T_{\text {conv }} \leq \sum_{k}\left(T\left(R_{k}\right)-T\left(R_{k-1}\right)\right) T^{*} / \gamma$, which implies (5.8).

Another acceleration type corresponds to the choice

$$
\mu_{M}(\vec{\sigma})=\frac{\gamma R_{n_{R}(\vec{\sigma})}}{T\left(R_{n_{R}(\vec{\sigma})}\right)}, \gamma>0 .
$$

This choice of $\mu$ is less demanding, and looks natural. It is exactly the value needed to provide for the convergence to zero in the time $T\left(R_{n_{R}(\vec{\sigma})}\right) T^{*} / \gamma$. Thus it would provide for the needed transient time, provided it would be kept constant and $T^{*}=\gamma$. In such a case instead of slowing down while approaching the SM the system would enter it in a very stressful manner and reveal strong chattering afterwards. Instead, due to (5.7), the acceleration law (5.9) features the same chattering level as the unaccelerated system.

Theorem 2. Under the conditions of Theorem 1 and the control (4.6), (4.7), (4.16), (5.5), (5.9) the convergence time satisfies the inequality

$$
T_{\text {conv }}(t) \leq T^{*} \sum_{k \leq n_{R}(\vec{\sigma}(t, x(t))} \max \left(1, \frac{T\left(R_{k}\right)}{\gamma R_{k}}\right)\left(R_{k}-\frac{T_{*}}{T^{*}} R_{k-1}\right) .
$$

Proof

The maximal time $\Delta t_{k}$ needed to reach the inner ball $\|\vec{\sigma}\|_{h} \leq R_{k-1}$ from the sphere $\|\vec{\sigma}\|_{h}=$ $R_{k}$ does not exceed the maximal convergence time from $\|\vec{\sigma}\|_{h} \leq R_{k-1}$ minus the minimal convergence time from $\|\vec{\sigma}\|_{h} \leq R_{k-1}$. I.e. $\Delta t_{k} \leq\left(T^{*} R_{k}-T_{*} / \mu^{\frac{\underline{T}(\underline{r}-1)}{\underline{r}}} R_{k-1}\right) / \mu$, provided $\mu$ remains constant. Hence this estimation is true until the first entrance into $\|\vec{\sigma}\|_{h} \leq R_{k-1}$. Thus, taking (5.9), (5.7) and $\mu \geq 1$ into account get $\Delta t_{k} \leq \min \left(1, \frac{T\left(R_{k}\right)}{\gamma R_{k}}\right)\left(T^{*} R_{k}-T_{*} R_{k-1}\right)$.

Note that without acceleration, i.e. for $\mu=1$, due to Lemma 1 the convergence time is roughly proportional to the current value of $\|\vec{\sigma}\|_{h}$ (for example, see the simulation results) [27, 35]. Thus, one naturally considers convergence-rate functions satisfying $\lim _{R \rightarrow \infty}(T(R) / R)=0$.

Fixed-time convergence cannot be assured if (5.9) is chosen. With an exponential sequence $\frac{R_{k-1}}{R_{k}}=$ const $<1$ get $1-\frac{T_{*} R_{k-1}}{T^{*} R_{k}}=$ const $<1$. Then over large distances one gets $n(\vec{\sigma})=$ 
$\mathcal{O}\left(\ln \|\vec{\sigma}\|_{h}\right)$. Let now $\lim _{R \rightarrow \infty} T(R)=T_{\text {max }}<\infty$. Then (5.10) implies that

$$
T_{\text {conv }}=\mathcal{O}\left(\int_{0}^{\ln \|\vec{\sigma}\|_{h}} T_{\max } d R\right)=\mathcal{O}\left(\ln \|\vec{\sigma}\|_{h}\right)
$$

I.e. the combination of FT and globally exponential convergence is obtained.

\subsection{Acceleration of quasi-continuous SMC}

Quasi-continuous MIMO SMC requires significant coordination of the inputs in order to avoid partial SMs before the overall SM $\vec{\sigma} \equiv 0$ is established. The decoupled closed-loop inclusion (5.3) is not possible. As in (4.17), let

$$
v_{i}=\alpha \mu^{\bar{r}} V_{i}\left(\vec{\sigma}_{i} \mu^{-\bar{r}}\right)=-\alpha \mu^{\bar{r}} \Theta_{\theta}\left(\frac{\left\|\vec{\sigma}_{i} \mu^{-\bar{r}}\right\|_{h}}{\left\|\vec{\sigma} \mu^{-\bar{r}}\right\|_{h}}\right) \operatorname{sat}\left(\eta \tilde{\Psi}_{i, r_{i}}\left(\vec{\sigma}_{i} \mu^{-\bar{r}}\right)\right)
$$

Instead of (5.4) we get

$$
\sigma_{i}^{\left(r_{i}\right)} \in \alpha \mu^{\bar{r}}\left([-C, C]+\left[K_{m}, K_{M}\right]\left(\left[-p_{0}, p_{0}\right]-\Theta_{\theta}\left(\frac{\left\|\vec{\sigma}_{i} \mu^{-\bar{r}}\right\|_{h}}{\left\|\vec{\sigma} \mu^{-\bar{r}}\right\|_{h}}\right) \operatorname{sat}\left(\eta \tilde{\Psi}_{i, r_{i}}\left(\vec{\sigma}_{i} \mu^{-\bar{r}}\right)\right)\right)\right)
$$

Now, using $\operatorname{deg} \tilde{\Psi}_{i, r_{i}}=0$, denote $\mu_{i}=\mu^{\frac{\bar{r}}{r_{i}}}, \mu_{i}^{r_{i}}=\mu^{\bar{r}}$, and get

$$
\begin{aligned}
\sigma_{i}^{\left(r_{i}\right)} \in & \alpha \mu_{i}^{r_{i}}\{[-C, C] \\
& +\left[K_{m}, K_{M}\right]\left(\left[-p_{0}, p_{0}\right]-\tilde{\Theta}_{i}(\vec{\sigma}) \operatorname{sat}\left(\eta \tilde{\Psi}_{i, r_{i}}\left(\sigma_{i}, \dot{\sigma}_{i} \mu_{i}^{-1}, \ldots, \sigma_{i}^{\left(r_{i}-1\right)} \mu_{i}^{-r_{i}+1}\right)\right\}\right. \\
\tilde{\Theta}_{i}(\vec{\sigma})= & \Theta_{\theta}\left(\frac{\mu_{i}\left\|\left(\sigma_{i}, \dot{\sigma}_{i} \mu_{i}^{-1}, \ldots, \sigma_{i}^{\left(r_{i}-1\right)} \mu_{i}^{-r_{i}+1}\right)\right\|_{h}}{\sum_{j=1}^{m} \mu_{j}\left\|\left(\sigma_{j}, \dot{\sigma}_{j} \mu_{j}^{-1}, \ldots, \sigma_{j}^{\left(r_{j}-1\right)} \mu_{j}^{-r_{j}+1}\right)\right\|_{h}}\right), \mu_{i}=\mu^{\frac{\bar{r}}{r_{i}}}, i=1, \ldots, m .
\end{aligned}
$$

The only difference of (5.4) and (5.12) is the presence of the control multiplier $\tilde{\Theta}_{i}$, which prevents the decoupling.

The procedure still leads to simple dynamics (3.1) under the following additional assumption. New assumption: all partial relative degrees coincide: $r_{1}=\ldots=r_{m}=\underline{r}=\bar{r}$.

That assumption is often true for mechanical systems (often $r_{i}=2[12,48,49]$ ).

Lemma 4. Let $\mu \geq 1, r_{1}=\ldots=r_{m}=\bar{r}$, then control (4.7), (5.11) provides for the convergence of any trajectory of system (5.12) starting in the region $\|\vec{\sigma}\|_{h} \leq 1$ to the $r$-sliding mode $\vec{\sigma}=0$ in some time belonging to $\left[T_{*} / \mu^{\bar{r}}, T^{*} / \mu\right]$.

Proof

Since $\mu_{1}=\ldots=\mu_{m}$, the lemma directly follows from (5.12) and Lemma 2 .

Now the results of Theorems 1, 2 are reclaimed. The resulting accelerated control is technically speaking not quasi-continuous. Indeed, it is not defined by a functional feedback. Due to the switching logic (5.7) it has the additional discrete dynamics of $\mu$. The following is the analogue of Theorem 1.

Theorem 3. Let all partial relative degrees be equal, and let $T_{\text {conv }}(t)$ be the convergence time to the $S M \sigma \equiv 0$ from the trajectory point $(t, x(t))$ of system (4.1) under the control (4.6), (4.7), (4.17), (5.11),(5.5), (5.6), (5.7). Then along the trajectory it satisfies the inequality (5.8)

$$
T_{\text {conv }}(t) \leq \frac{T^{*}}{\gamma} T\left(R_{n_{R}(\vec{\sigma}(t, x(t)))}\right) .
$$

Each transient trajectory starting at $\vec{\sigma}_{0}=\vec{\sigma}\left(t, x\left(t_{0}\right)\right)$ exactly features the number $n_{R}\left(\vec{\sigma}_{0}\right)-1$ of control jumps caused by the jumps of $\mu$ (5.7). 
It is possible to get rid of the control discontinuities (5.7), which take place each time the trajectory $(t, x(t))$ (or $\vec{\sigma}(t)$ ) for the first time enters the region $\|\vec{\sigma}\|_{h} \leq R_{k}, k=1,2, \ldots$ One of the simplest ways is to replace the discontinuous signal $\mu(t)$ in control with a locally Lipschitzian function $\hat{\mu}(t)$ which tracks the piece-wise constant function $\mu(t)$.

The switch of $\mu$ from $\mu_{M}\left(R_{k+1}\right)$ to $\mu_{M}\left(R_{k}\right)$ will cause the corresponding transient of $\hat{\mu}$. Choose any $\gamma_{0}>1, \gamma_{1}>0$. Require that during this transient the system does not leave the region $\|\vec{\sigma}\|_{h} \leq \gamma_{0} R_{k}$. Also this internal transient time should not exceed $\gamma_{1}\left(T\left(R_{k}\right)-T\left(R_{k-1}\right)\right)$ and should be over before the system trajectory enters the inner region $\|\vec{\sigma}\|_{h} \leq R_{k-1}$.

Due to the chosen homogeneity degree -1 of the DI (4.2) any smooth homogeneous norm along the trajectory features the Lipschitz inequality $\left|\left\|\vec{\sigma}\left(t_{1}, x\left(t_{1}\right)\right)\right\|_{h}-\left\|\vec{\sigma}\left(t_{2}, x\left(t_{2}\right)\right)\right\|_{h}\right| \leq$ $\left(L_{0}+L_{1} \sup \left\|\sigma^{(r)}\right\|\right)\left|t_{2}-t_{1}\right|$ for some $L_{0}, L_{1}>0$ to be found in advance (Lemma 1$)$. Therefore, calculate the maximal time $\Delta_{t}(k)$ available for the switching of $\mu$ as

$$
\Delta_{t}(k)=\min \left\{\frac{\min \left[\left(\gamma_{0}-1\right) R_{k}, R_{k+1}-R_{k}\right]}{L_{1}\left[2 K_{M} \alpha \mu_{M}^{\bar{r}}\left(R_{k+1}\right)+C\right]+L_{0}}, \gamma_{1}\left(T\left(R_{k}\right)-T\left(R_{k-1}\right)\right)\right\} .
$$

Since $\mu \leq \max \left[1, \mu_{M}\left(R_{k+1}\right)\right]$ holds due to (5.6), (5.7), $|\dot{\hat{\mu}}| \geq \max \left[1, \mu_{M}\left(R_{k+1}\right)\right] / \Delta_{t}(k)$ should satisfy the above transient restrictions. The resulting control law is

$$
\begin{aligned}
& v_{i}=\alpha \hat{\mu}^{\bar{r}} V_{i}\left(\vec{\sigma}_{i} \hat{\mu}^{-\bar{r}}\right) ; \hat{\mu}\left(t_{0}\right)=\mu\left(t_{0}\right), \\
& \dot{\hat{\mu}}=-\alpha_{\mu}(\vec{\sigma}) \operatorname{sign}(\hat{\mu}-\mu), \alpha_{\mu}=\max \left[1, \mu_{M}\left(R_{n_{R}(\vec{\sigma})+1}\right)\right] / \Delta_{t}\left(n_{R}(\vec{\sigma})\right) .
\end{aligned}
$$

Any larger function $\alpha_{\mu}(\vec{\sigma})$ is also acceptable if it is Lebesgue measurable and locally essentially bounded.

Theorem 4. Under the conditions of Theorem 3 let $\gamma_{0}>1, \gamma_{1}>0$, then the control (4.6), (4.7), (4.17), (5.11),(5.5), (5.6), (5.7), (5.13), (5.14) provides for the FT convergence to $r$-SM $\sigma=0$. The convergence time along the trajectory of (4.1) satisfies the inequality

$$
T_{\text {conv }}(t) \leq\left(\frac{T^{*} \gamma_{0}}{\gamma}+\gamma_{1}\right) T\left(R_{n_{R}(\vec{\sigma}(t, x(t)))}\right) .
$$

The control remains continuous until the very entrance into the $S M \sigma \equiv 0$.

Proof

The trajectories of the DI (5.12) starting from the homogeneous ball $\|\vec{\sigma}\|_{h} \leq \gamma_{0}$ with $\hat{\mu}=1$ stabilize at $\vec{\sigma}=0$ in time not exceeding $\gamma_{0} T^{*}$. Respectively the trajectories $\vec{\sigma}(t)$ starting from the ball $\|\vec{\sigma}\|_{h} \leq \gamma_{0} R$ will stabilize in the time $\gamma_{0} T^{*} R / \hat{\mu}$ provided $\hat{\mu}=$ const $\geq 1$.

Let $\mu_{M}\left(R_{k}\right)>1$. The opposite case is similarly considered. When $\vec{\sigma}(t)$ enters the ball $\|\vec{\sigma}\|_{h} \leq R_{k}$ for the first time, the function $\mu(t)$ takes on the locally constant value (5.6) in a jump from its previous value $\mu_{M}\left(R_{k+1}\right)$. The function $\hat{\mu}$ remains continuous and starts to move from $\mu_{M}\left(R_{k+1}\right)$ to the new value $\mu_{M}\left(R_{k}\right)$. The time (5.13) is required for it to stabilize at the new value (5.6). Due to (5.13) during this time $\vec{\sigma}$ does not leave the larger ball $\|\vec{\sigma}\|_{h} \leq \gamma_{0} R_{k}$ and does not enter the smaller ball $\|\vec{\sigma}\|_{h} \leq R_{k-1}$. After that $\hat{\mu} \equiv \mu \equiv \mu_{M}\left(R_{k}\right)$ is kept. Respectively it takes not more than $\left(T\left(R_{k}\right)-T\left(R_{k-1}\right)\right) \frac{\gamma_{0} T^{*}}{\gamma}$ for the trajectory $\vec{\sigma}(t)$ to reach the next homogeneous ball $\|\vec{\sigma}\|_{h} \leq R_{k-1}$ for the first time. Thus, the total time $\Delta t_{k}$ required to reach $\|\vec{\sigma}\|_{h} \leq R_{k-1}$ from $\|\vec{\sigma}\|_{h} \leq R_{k}$ is estimated as $\Delta t_{k} \leq\left(\frac{\gamma_{0} T^{*}}{\gamma}+\gamma_{1}\right)\left(T\left(R_{k}\right)-T\left(R_{k-1}\right)\right)$.

In practice one just takes $L_{0}, L_{1}$ sufficiently large in (5.13). The alternative switching (5.9) of $\mu$ can be similarly modified to avoid discontinuities.

Note that though $r_{1}=\ldots=r_{m}$ is assumed in Theorems 3,4 , the acceleration procedures of this Section are still applicable to a MIMO system with unequal partial relative degrees.

Indeed, in that case due to (5.12) each output component $\sigma_{i}$ has its own acceleration rate. Though the MIMO control becomes quasi-continuous between the $\mu$ switchings, the system is not anymore one uniformly accelerated system for each constant $\mu$. It can happen that there is no $\theta>0$ providing for the overall-system FT stability for any $\mu \geq 1$. Nevertheless, if the 
acceleration factor $\mu$ is a priori bounded, $\mu \leq \mu_{\max }$, then for sufficiently small $\theta>0$ the FT convergence is preserved over the whole finite range of $\mu$.

Thus, one definitely can use the approach over compact regions of initial conditions, or guarantee the boundedness of $\mu$ by some saturation mechanism (see the simulation results). The acceleration procedure can be considered as a valuable practical design tool for any homogeneous FT stable system.

\subsection{Output-feedback SM acceleration}

Let $u=G^{-1} v, v_{i}=\alpha \mu^{\bar{r}} V_{i, \mu}(\vec{\sigma})$ be the resulting accelerated control with the addition of the $\mu$ definition (5.7) and (5.6) or (5.9). Between its jumps $\mu$ remains constant, which allows easy application of the differentiators. Thus the output-feedback control gets the form

$$
\begin{aligned}
& u=G^{-1}(t, x) v, v_{i}=\alpha \mu^{\bar{r}} V_{i, \mu}(z), z=\left(z_{1}, z_{2}, \ldots, z_{m}\right), i=1, \ldots, m \\
& \dot{z}_{i}=D_{r_{i}-1}\left(z_{i}, \sigma_{i}, L\right), L=\gamma_{L}\left(K_{M}\left(1+p_{0}\right) \alpha+C\right) \mu^{\bar{r}}, \gamma_{L} \geq 1,
\end{aligned}
$$

in combination with (5.7) and (5.6) or (5.9). Thus $L$ switches together with $\mu$, any $\gamma_{L} \geq 1$ can be taken. From the moment as the differentiators have converged for the first time, they remain in the steady state forever.

In the case when the actual parameter which is fed to the control, is $\hat{\mu}$, whereas $\hat{\mu}$ tracks the discontinuous signal $\mu$, one should switch $L$ to the next lower value only at the moment when $\hat{\mu}$ has stabilized at the new value, i.e. with the precalculated delay (5.13) after the switch of $\mu$.

In practice, in the presence of noises and sampling periods the differentiator error is proportional to $L$, but $L$ is a piece-wise constant non-increasing function of time. Thus after each switch of $L$ an infinitesimal transient is possible to the new smaller steady-state errors. At any moment the overall system with any fixed $\mu \geq 1$ is FT stable, the piece-wise constant $\mu$ monotonously decreases to 1 when the trajectory approaches $\vec{\sigma}=0$. Thus the system cannot lose its FT stability even if due to some external short-time disturbance the trajectory performs an unpredictable jump.

The only remaining question is how to initiate the differentiators. There are many possible ways, but probably the simplest one is to roughly calculate the initial derivatives values by divided differences with some sampling step taking into account the possible presence of noises (see Section 7). One can also provide some small additional time for the initial differentiator convergence before applying the control for the first time.

Since the system remains homogeneous in some vicinity of $\vec{\sigma}=0$, for any bounded set of initial conditions the accuracy in the presence of sufficiently small noises, switching and sampling delays remains the same as of the original system. The accuracy $\left|\sigma_{i}^{(j)}\right| \leq \omega_{i, j} \delta^{r_{i}-j}$ is established in FT, where $\delta$ is calculated as $\delta=\max \left(\delta_{t}, \max _{i} \delta_{0, i}^{1 / r_{i}}\right)$ for sampling time periods not exceeding $\delta_{t}>0$ and the maximal possible sampling error $\delta_{0, i} \geq 0$ of $\sigma_{i}$ [27].

\section{ACCELERATED STABILIZATION AT EQUILIBRIUM}

The same technique can be used for the FT stabilization by a continuous feedback of a smooth dynamic system at its equilibrium.

\subsection{Stabilization problem}

Once more consider dynamic system (4.1). Suppose that the open-loop system has an equilibrium point $P \in \mathbb{R}^{n}$, i.e. $a(t, P)=0$, and also $\sigma(t, P)=0$. The task is to stabilize the system at the equilibrium $P$ by continuous control.

The main assumptions of Section 4.1 are preserved. In particular, the system is assumed to have the relative degree $r$. This time it is supposed to have no zero dynamics. It means that $r=\left(r_{1}, \ldots, r_{m}\right), r_{1}+\ldots+r_{m}=n$. It also means that the successive total time derivatives 
$\sigma_{i}^{(j)}$ with $j=0,1, \ldots, r_{i}-1, i=1, \ldots, m$, vanish at $P$, and can be used as new coordinates [23]. Respectively we do not further distinguish $x$ and $\vec{\sigma}$.

In particular, (4.1) coincides with (4.2) and takes on the form

$$
\sigma^{(r)}=h(t, \vec{\sigma})+g(t, \vec{\sigma}) u,
$$

where $h(t, \vec{\sigma})=\left(h_{1}(t, \vec{\sigma}), \ldots, h_{m}(t, \vec{\sigma})\right)^{T}, h(t, 0)=0$, and nonsingular $g(t, \vec{\sigma})$ are unknown and smooth. Solutions of (6.1) are assumed extendable till infinity in time, provided $\|G u\| /\|\vec{\sigma}\|$ is bounded by a constant along the trajectory.

While the assumptions on $g$ are preserved, assumptions on $h$ are to change. The function $h$ is assumed to have some continuous upper norm bound

$$
\|h(t, \vec{\sigma})\| \leq \Phi(\vec{\sigma}), \Phi(0)=0 .
$$

We also assume that $\|\Phi(\vec{\sigma})\| /\|\vec{\sigma}\|$ is uniformly bounded, which is consistent with the above assumption on the indefinite extension of solutions in time and smoothness of the system at its equilibrium.

Introduce some arbitrary parameter $q>0$ and let the coordinates $\sigma_{i}, \ldots, \sigma_{i}^{\left(r_{i}-1\right)}$ have the homogeneity weights $r_{i}+q, r_{i}+q-1, \ldots, 1+q$ respectively. The corresponding dilation is

$$
\begin{aligned}
& d_{\kappa} \vec{\sigma}=\left(d_{1, \kappa} \vec{\sigma}_{1}, \ldots, d_{m, \kappa} \vec{\sigma}_{m}\right), \kappa>0, \\
& d_{i, \kappa} \vec{\sigma}_{i}=\left(\kappa^{r_{i}+q} \sigma_{i}, \ldots, \kappa^{1+q} \sigma_{i}^{\left(r_{i}-1\right)}\right) .
\end{aligned}
$$

The corresponding homogeneous norms have the form

$$
\begin{aligned}
& \left\|\vec{\sigma}_{i}\right\|_{h}=\left(\left|\sigma_{i}\right|^{\frac{\bar{\rho}_{i}}{r_{i}+q}}+\left|\dot{\sigma}_{i}\right|^{\frac{\bar{\rho}_{i}}{\bar{r}_{i}+q-1}}+\ldots+\left|\sigma_{i}^{\left(r_{i}-1\right)}\right|^{\frac{\bar{\rho}_{i}}{1+q}}\right)^{\frac{1}{\bar{\rho}_{i}}}, \bar{\rho}_{i}>0, \\
& \|\vec{\sigma}\|_{h}=\left\|\sigma_{1}\right\|_{h}+\ldots+\left\|\sigma_{m}\right\|_{h} .
\end{aligned}
$$

6.1.1. Homogeneous SISO FT stabilization. In the SISO case $m=1, \sigma, u \in \mathbb{R}, r \in \mathbb{N}, g=G=$ $\pm 1, \Delta g=0$. Naturally $g=1, u=v$ are taken. The homogeneity weights of the coordinates $\vec{\sigma}$ are $\operatorname{deg} \sigma=r+q, \operatorname{deg} \dot{\sigma}=r+q-1, \ldots, \operatorname{deg} \sigma^{(r-1)}=1+q$, and respectively $\operatorname{deg} \sigma^{(r)}=q$, $q>0$. Obviously $[26,27],(4.6)-(4.8)$ become

$$
\sigma^{(r)} \in[-1,1] \Phi(\vec{\sigma})+\left[K_{m}, K_{M}\right] u .
$$

Local SISO stabilization. There are a lot of controllers of the form

$$
u=-\alpha N(\vec{\sigma}) \Psi_{r}(\vec{\sigma}),\left|\Psi_{r}(\vec{\sigma})\right| \leq 1, \alpha>0, \operatorname{deg} N=q, \operatorname{deg} \Psi_{r}=0,
$$

locally FT stabilizing the equation $\sigma^{(r)}=u$ for sufficiently large $\alpha[29,32]$. Here $N$ is any positive-definite continuous homogeneous function of the weight $q$. In particular, $N(\vec{\sigma})=\|\vec{\sigma}\|_{h}^{q}$ may be taken. The differential equation $\Psi(\vec{\sigma})=0$ is FT stable.

The following simple lemma explains the power of such controllers in the vicinity of the equilibrium.

Lemma 5. Let $C>0$ be any real number. Then the inequality

$$
|\Phi(\vec{\sigma})| \leq C N(\vec{\sigma})
$$

holds in a sufficiently small vicinity of $\vec{\sigma}=0$.

Proof

Choose the norm $\|\vec{\sigma}\|_{h}$ as in (6.4) for the SISO case $m=1$ and $\bar{\rho}_{1}=2 q$. The function $N^{1 / q}(\vec{\sigma})$ is also a homogeneous norm, therefore $\|\vec{\sigma}\|_{h} \leq C_{0} N^{1 / q}(\vec{\sigma})$ holds for some $C_{0}>0$. To see it one just needs to compare these two continuous positive-definite functions of the weight 1 on the 
homogeneous sphere $\|\vec{\sigma}\|_{h}=1$. Now the inequalities

$$
\|\vec{\sigma}\|=\left(\Sigma\left|\sigma^{(j)}\right|^{2}\right)^{\frac{1}{2}} \leq C C_{0}^{-q}\left(\sum\left|\sigma^{(j)}\right|^{\frac{2 q}{r-j+q}}\right)^{\frac{1}{2}}=C C_{0}^{-q}\|\vec{\sigma}\|_{h}^{q} \leq C N(\vec{\sigma})
$$

are valid in a vicinity of 0 and finish the proof.

Controllers (6.6) can be built on the basis of homogeneous quasi-continuous controllers from $[28,37]$. Once more the simplest such controller is the "polynomial" controller [10]

$$
\Psi_{r}=\frac{\left\lfloor\sigma^{(r-1)}\right\rceil^{\frac{\rho_{0}}{1+q}}+\beta_{r-2}\left\lfloor\sigma^{(r-1)}\right\rceil^{\frac{\rho_{0}}{2+q}}+\ldots+\beta_{r-2}\left\lfloor\left.\sigma\right|^{\frac{\rho_{0}}{r+q}}\right.}{\left|\sigma^{(r-1)}\right|^{\frac{\rho_{0}}{1+q}}+\beta_{r-2}\left|\sigma^{(r-1)}\right|^{\frac{\rho_{0}}{2+q}}+\ldots+\beta_{r-2}|\sigma|^{\frac{\rho_{0}}{r+q}}} .
$$

Any $\rho_{0}>0$ can be taken, $\beta_{j}>0$. The corresponding parametric sets $\left\{\beta_{0}, \ldots, \beta_{r-2}\right\}$ are arbitrary for $r=1,2$. Note that $\Psi_{r}$ is $l$ times continuously differentiable if $\rho_{0}>l+r+q$ and $\vec{\sigma} \neq 0$.

Any controller (6.6) locally stabilizes (6.5) for sufficiently large $\alpha$, thus, solving the problem for any natural number $r$ and $K_{m}, K_{M}$. Indeed, since $|\Phi(\vec{\sigma})| /|| \vec{\sigma} \|$ is bounded, due to Lemma 5 inequality (6.7) holds in some vicinity of 0 for any $C>0$. All solutions of the closed system (6.1), (6.6) locally satisfy the homogeneous inclusion

$$
\sigma^{(r)} \in[-C, C] N(\vec{\sigma})-\alpha\left[K_{m}, K_{M}\right] N(\vec{\sigma}) \Psi(\vec{\sigma}) .
$$

Solutions of the latter inclusion approximately satisfy the FT stable differential equation $\Psi_{r}(\vec{\sigma})=0$ for sufficiently large $\alpha[29]$. The FT stability of (6.9) follows from the robustness of the FT stability with respect to homogeneous disturbances [27].

Global SISO stabilization. If the restriction (6.7) holds globally the same controller (6.6) will provide for the global FT stabilization. Note that condition (6.7) is mildly restrictive, since $N(\vec{\sigma}) \rightarrow \infty$ for $\|\vec{\sigma}\| \rightarrow \infty$. Otherwise the system is globally stabilized by the non-homogeneous control

$$
u=-\alpha(N(\vec{\sigma})+\|\vec{\sigma}\|) \Psi_{r}(\vec{\sigma})
$$

for sufficiently large $\alpha$.

6.1.2. Homogeneous MIMO FT stabilization. Similarly to the MIMO SMC apply the control transformation (4.6) and let

$$
v_{i}=\alpha V_{i}(\vec{\sigma}), i=1, \ldots, m,
$$

where the magnitudes of $V_{i}$ coincide. Choose some FT stabilizing control $-\alpha N_{i}\left(\vec{\sigma}_{i}\right) \tilde{\Psi}_{i}\left(\vec{\sigma}_{i}\right)$ for each component of $\sigma$ like in Section 6.1.1. Respectively, the homogeneous MIMO stabilization control is defined by

$$
\begin{aligned}
& V_{i}(\vec{\sigma})=-N(\vec{\sigma}) \Theta_{\theta}\left(\xi_{i}\right) \operatorname{sat}\left(\eta_{i} \tilde{\Psi}_{i, r_{i}}\left(\vec{\sigma}_{i}\right), \eta_{i} \geq 1,0 \leq \theta \leq 1, \xi_{i}=\left\|\vec{\sigma}_{i}\right\|_{h} /\|\vec{\sigma}\|_{h},\right. \\
& N(\vec{\sigma})=\max \left\{N_{1}\left(\vec{\sigma}_{1}\right), \ldots, N_{m}\left(\vec{\sigma}_{m}\right)\right\} .
\end{aligned}
$$

Lemma 5 holds also in the MIMO case. Thus, similarly to the MIMO SM case, control (6.10), (6.11) is continuous and provides for the local FT stabilization for any sufficiently large $\alpha$ and sufficiently small $\theta$ [32]. The convergence is global provided (6.7), $\|\Phi(\vec{\sigma})\| \leq C N(\vec{\sigma})$, holds globally.

\subsection{Accelerated MIMO FT stabilization}

The following procedure allows voluntary convergence rate regulation. Once more we assume that $r_{1}=r_{2}=\ldots=r_{m}=\bar{r}$. The accelerated control analogue to (5.11) gets the form

$$
\begin{aligned}
& v_{i}=\alpha \mu^{\bar{r}} V_{i}\left(\Lambda_{\mu} \vec{\sigma}\right)=-\alpha \mu^{\bar{r}} \Theta_{\theta}\left(\frac{\left\|\Lambda_{\mu} \vec{\sigma}_{i}\right\|_{h}}{\left\|\Lambda_{\mu} \vec{\sigma}\right\|_{h}}\right) N\left(\Lambda_{\mu} \vec{\sigma}\right) \operatorname{sat}\left(\eta \tilde{\Psi}_{i, r_{i}}\left(\Lambda_{\mu} \vec{\sigma}\right)\right), \\
& \Lambda_{\mu} \vec{\sigma}_{i}=\left(\sigma_{i}, \mu^{-1} \dot{\sigma}_{i}, \ldots, \mu^{-\bar{r}+1} \sigma_{i}^{(\bar{r}-1)}\right), \Lambda_{\mu} \vec{\sigma}=\left(\Lambda_{\mu} \sigma_{1}, \ldots, \Lambda_{\mu} \sigma_{m}\right),
\end{aligned}
$$


where $\mu$ satisfies (5.5), (5.6), (5.7).

Note that $\operatorname{deg} N=q, \quad N \geq 0$ imply that $\mu^{\bar{r}} N\left(\Lambda_{\mu} \vec{\sigma}\right)=N\left(\ldots, \mu^{\bar{r}(\bar{r}-j+q) / q-j} \sigma_{i}^{(j)}, \ldots\right)=$ $N\left(\ldots, \mu^{(\bar{r}-j)(\bar{r} / q+1)} \sigma_{i}^{(j)}, \ldots\right)$ and $c_{1}\|\vec{\sigma}\|_{h}^{q} \leq N(\vec{\sigma}) \leq c_{2}\|\vec{\sigma}\|_{h}^{q}$ hold for any $\vec{\sigma}, \mu>0$ and some $c_{1}, c_{2}>0$. Now $\mu \geq 1$ and (6.4), (6.7) imply $\|\Phi(\vec{\sigma})\| \leq \hat{C} \mu^{\bar{r}} N\left(\Lambda_{\mu} \vec{\sigma}\right)$ for some $\hat{C} \geq C$.

Theorem 5. Let all partial relative degrees be equal, (6.7) hold globally, and let $T_{\text {conv }}(t)$ be the desired convergence time to the equilibrium $\sigma \equiv 0$ from the trajectory point $(t, x(t))$, under the control (4.6), (5.5), (5.6), (5.7), (6.12). Then the inequality $T_{\text {conv }}(t) \leq \frac{T^{*}}{\gamma} T\left(R_{n_{R}(\vec{\sigma}(t, x(t)))}\right)$ holds along the trajectory for sufficiently large $\alpha>0$. Each transient trajectory starting at $\vec{\sigma}_{0}=\vec{\sigma}\left(t_{0}, x\left(t_{0}\right)\right)$ exactly features the number $n_{R}\left(\vec{\sigma}_{0}\right)-1$ of control jumps caused by the jumps of $\mu$ (5.7). At any other time the control remains continuous.

Once more the control discontinuity is avoided by introducing the variable $\hat{\mu}$ tracking the piece-wise constant function $\mu(t)$. Any differentiable homogeneous norm $\|\vec{\sigma}\|_{h}$ has a Lipschitz constant equal to $L_{0}+L_{1}\left[2 K_{M} \alpha \mu_{M}^{\bar{r}}\left(R_{k+1}\right)+C\right]$, where $L_{0}, L_{1}$ are calculated for the homogeneous DI $\sigma_{i}^{(\bar{r})} \in N_{M}\left(\|\vec{\sigma}\|_{h}\right)[-1,1], i=1, \ldots, m, N_{M}(\rho)=\max _{\|\vec{\sigma}\|_{h} \leq \rho} N(\vec{\sigma})$ (Lemma 1).

Theorem 6. Under the conditions of Theorem 5 let $\gamma_{0}>1, \gamma_{1}>0$, then the control (4.6), (5.5), (5.6), (5.7), (5.13), (5.14) using the function $V$ from (6.11) provides for the FT convergence to the $S M \sigma \equiv 0$. The convergence time along the trajectory $(t, x(t))$ of (4.1) satisfies the inequality (5.15). The control remains continuous all the time.

The proofs are exactly the same as for the SMC acceleration. Fixed-time convergent systems can be very sensitive to sampling errors and delays at large initial conditions [33]. Nevertheless, the accelerated system demonstrates the well-known accuracy of the original, not accelerated system for any compact set of initial conditions if the noises and delays are small enough.

Hence the accuracy $\left|\sigma_{i}^{(j)}\right| \leq \omega_{i, j} \delta^{\bar{r}-j+q}$ is established in FT for sufficiently small $\delta$. Here $\delta=\max \left(\delta_{t}, \max _{i, j} \delta_{i, j}^{1 / \bar{r}}\right)$ is calculated for the maximal possible sampling time period $\delta_{t} \geq 0$ and the maximal possible sampling error $\delta_{i, j} \geq 0$ of $\sigma_{i}^{(j)}, j=1, \ldots, \bar{r}, i=1,2, \ldots, m[27,35]$.

Output feedback stabilization. Robust exact differentiators [26] can be applied over any compact region of initial conditions. In that case the highest derivatives $\sigma_{i}^{(\bar{r})}$ are bounded by some constants to be roughly estimated in advance, and the exact robust derivative estimations are produced. A single $(\bar{r}-1)$ th-order differentiator is to be applied to each output $\sigma_{i}$.

Global output feedback requires usage of the differentiators [36] with variable parameter $L$. Between its jumps $\mu$ remains constant, which allows easy application of such differentiators. The required uniform boundedness of $\dot{L} / L$ is trivial here.

\section{SIMULATION RESULTS}

MIMO car control. Demonstrate that quasi-continuous control acceleration over a compact operational region does not require $r_{1}=\ldots=r_{m}$. Consider a simple MIMO ("bicycle") model of car control [46]

$$
\begin{aligned}
& \dot{x}=V \cos \varphi, \dot{y}=V \sin \varphi, \dot{\varphi}=\frac{V}{\Delta} \tan \hat{\theta} \\
& \dot{V}=\mu_{1} T_{n e t}(V, \rho)-\mu_{2} V^{2}-\mu_{3} R_{x}, \mu_{3} R_{x}=\varepsilon(1-\cos (5 \hat{\theta})), \\
& \mu_{1} T_{n e t}(V, \rho)=(2.5 \sin \rho-0.7)\left(1-0.001(V-9)^{2}\right), \\
& \dot{\hat{\theta}}=u_{1}, \dot{\rho}=u_{2}
\end{aligned}
$$

where $x$ and $y$ are Cartesian coordinates of the rear-axle middle point, $\varphi$ is the orientation angle, $V$ is the longitudinal velocity, $\Delta$ is the length between the two axles and $\hat{\theta}$ is the steering angle (i.e. the first real input) (Fig. 7.1), $T_{n e t}(V, \rho)$ is the net combustion torque of the engine, $\rho$ is the throttle angle (i.e. the second real input). The saturation of controls is introduced: $\rho \in[0,1.05], \hat{\theta} \in[-1.2,1.2] . R_{x}$ is the rolling resistance of the tires. Parameters $\mu_{2}=0.005$, 
$\Delta=5 \mathrm{~m}$ were taken. For simplicity brakes are not applied. Usually $T_{n e t}$ is available as a table function of the engine angle velocity and $\rho$. It is presented here by some regression roughly approximating the data from [46], Fig. 9-6. The rolling resistance is voluntarily represented here by a function, corresponding to some mechanical car damage, $\varepsilon=0.1$.

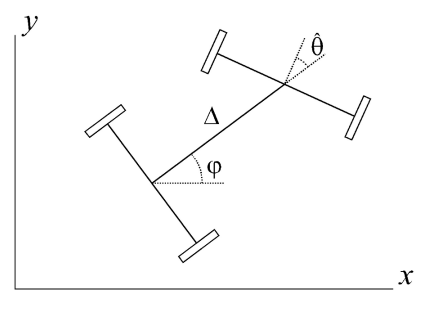

Figure 7.1: The car model.

The task is to steer the car from a given initial position and speed to the trajectory $y=y_{c}(x)$, and $V=V_{c}(t)$, where $x(t), y_{c}(x(t)), y(t)$ and $V_{c}(t)$ are assumed to be available in real time.

Define $\sigma_{1}=y-y_{c}(x), \sigma_{2}=V-V_{c}(t)$. The initial values are $V=4 m / s, x=y=\varphi=\rho=$ $\hat{\theta}=0$ at $t=0, y_{c}(x)=10 \sin (0.05 x)+5, V_{c}(t)=9+\sin (0.5 t)$.

In order to define $G$ calculate the matrix

$$
\begin{aligned}
& g=\left[\begin{array}{cc}
\dddot{\sigma}^{\prime} & \dddot{\sigma}^{\prime}{ }^{\prime} u_{1} \\
\ddot{\sigma}_{2 u_{1}}^{\prime} & \ddot{\sigma}_{2 u_{2}}^{\prime}
\end{array}\right] \\
& \dddot{\sigma}_{1, u_{1}}^{\prime}=\frac{V^{2}}{\Delta} \frac{\cos \varphi+y_{c}^{\prime} \sin \varphi}{\cos ^{2} \hat{\theta}}-5 \varepsilon V \sin (5 \hat{\theta})\left(\sin \varphi-y_{c}^{\prime} \cos \varphi\right) \text {, } \\
& \dddot{\sigma}_{1, u_{2}}^{\prime}=2.5 \cos \rho\left(1-0.001(V-9)^{2}\right)\left(\sin \varphi-y_{c}^{\prime} \cos \varphi\right) \text {, } \\
& \ddot{\sigma}_{2, u_{1}}^{\prime}=-5 \varepsilon V \sin (5 \hat{\theta}), \ddot{\sigma}_{2, u_{2}}^{\prime}=2.5 \cos \rho\left(1-0.001(V-9)^{2}\right) \text {; }
\end{aligned}
$$

and, taking into account that the mechanical damage should be considered unknown, define $G=\left[\begin{array}{cc}\frac{V^{2}}{\Delta} \frac{\cos \varphi}{\cos ^{2} \hat{\theta}} & 0 \\ 0 & 2.5 \cos \rho\end{array}\right], K_{m}=K_{M}=1$. Recall that $u=G^{-1} v$.

The relative degree of the system is $r=(3,2)$ and the quasi-continuous $(3,2)$-SM controllers solve the problem. The controller magnitude $\alpha$, the parameters $\eta_{1}$, and $\eta_{2}$ are conveniently found by simulation. It was taken $\eta_{1}=\eta_{2}=2, \alpha=10$, the differentiator parameters are $\lambda_{0}=1.1, \lambda_{1}=1.5, \lambda_{2}=3$. The differentiators are initiated by the divided differences with the sampling step 0.01 . The control is applied starting from $t_{0}=0.1$ in order to provide some additional time for the differentiators' convergence.

Define the homogeneous norms as in (4.10) with $\rho_{1}=r_{1}=3, \rho_{2}=r_{2}=2$. The outputfeedback controller [28] with the acceleration parameter $\mu$ is

$$
\begin{aligned}
& v_{1}=-10 \mu^{3} \Theta_{0.5}\left(\frac{\left\|z_{1} \mu^{-3}\right\|_{h}}{|| z \mu^{-3} \|_{h}}\right) \cdot \operatorname{sat}\left(2 \frac{z_{1,2} \mu^{-3}+0.3\left(\left|z_{1,1}\right| \mu^{-3}+0.4\left|z_{1,0} \mu^{-3}\right|^{\frac{2}{3}}\right)^{\frac{1}{2}} \frac{z_{1,1} \mu^{-3}+0.4\left\lfloor\left. z_{1,0} \mu^{-3}\right|^{\frac{2}{3}}\right.}{\left|z_{1,1}\right| \mu^{-3}+0.4\left|z_{1,0} \mu^{-3}\right|^{\frac{2}{3}}}}{\left|z_{1,2}\right| \mu^{-3}+0.3\left(\left|z_{1,1}\right| \mu^{-3}+0.4\left|z_{1,0} \mu^{-3}\right|^{\frac{2}{3}}\right)^{\frac{1}{2}}}\right), \\
& v_{2}=-10 \mu^{3} \Theta_{0.5}\left(\frac{\left\|z_{2} \mu^{-3}\right\|_{h}}{\left\|z \mu^{-3} \mid\right\|_{h}}\right) \operatorname{sat}\left(2 \frac{z_{2,1} \mu^{-3}+0.4\left\lfloor z_{2,0} \mu^{-3}\right\rceil^{\frac{1}{2}}}{\left|z_{2,1}\right| \mu^{-3}+0.4\left|z_{2,0} \mu^{-3}\right|^{\frac{1}{2}}}\right), \\
& \dot{z}_{1}=D_{2}\left(z_{1}, \sigma_{1}, 100 \mu^{3}\right), \quad \dot{z}_{2}=D_{1}\left(z_{2}, \sigma_{2}, 100 \mu^{3}\right) .
\end{aligned}
$$

The non-accelerated performance of the system corresponds to $\mu=1$ and is demonstrated in the left columns of Figs. 7.2, 7.3, 7.4.

The assumption $r_{1}=r_{2}$ does not hold here, which means that the acceleration procedure is not well established. Recall that in that case the acceleration is not uniform for different components, and there is possibly no $\theta \neq 0$, which would assure convergence of (5.12) for all 


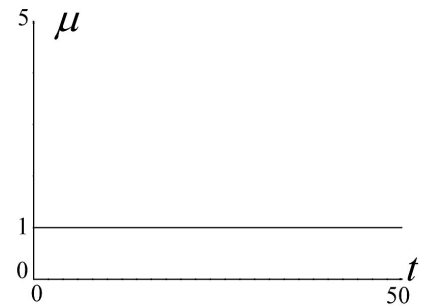

a.

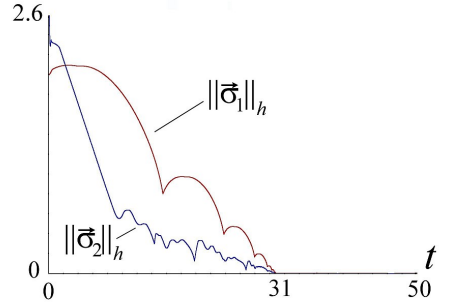

c.

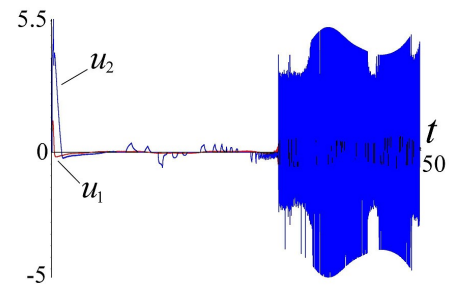

e.

no acceleration

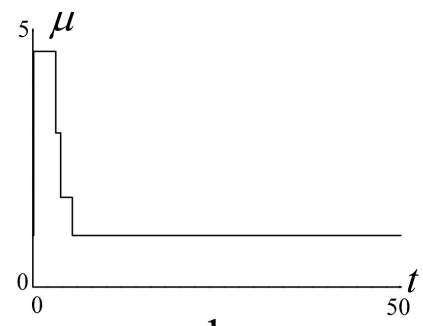

b.
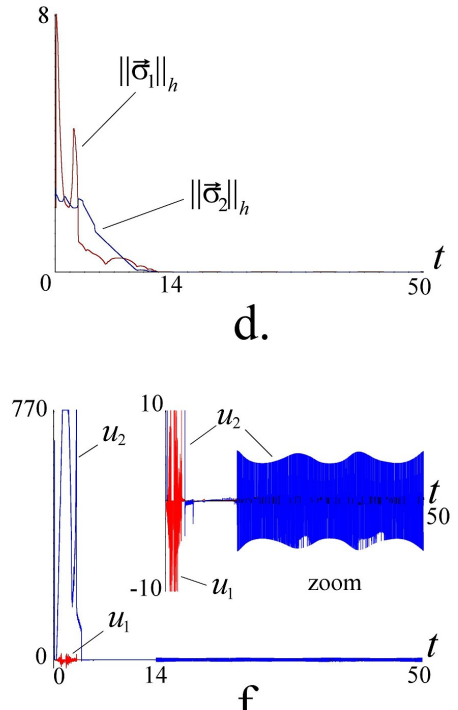

f.

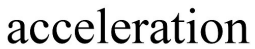

Figure 7.2: Comparison of the not accelerated (on the left) and accelerated (on the right) $(3,2)$-SM car control: acceleration factor $\mu$, homogeneous norms of the tracking errors $\vec{\sigma}_{1}, \vec{\sigma}_{2}$, quasi-continuous controls.

$\mu \geq 1$. One can still always find such value of $\theta$ for any compact range of $\mu$. In practice it corresponds to the local acceleration which still can lead to significant performance benefits.

The convergence-rate function $T(R)=3 \ln (R+1)$ is chosen with the sequence $R_{n}=$ $n$. Apply the acceleration. The initial acceleration parameter $\mu(0)=1$, and it takes the corresponding calculated value at $t=t_{0}=0.1$. It is clearly seen from Figs. 7.2, 7.3, 7.4 that significant transient acceleration is achieved, while preserving the "soft" approaching of the desired trajectory by the car. Practically the same accuracy is achieved with and without acceleration. The accuracies obtained for the Euler/sampling steps $\tau=10^{-4}, 10^{-5}$ are described by the component-wise inequalities

$$
\left.\begin{array}{l}
\left(\left|\sigma_{1}\right|,\left|\dot{\sigma}_{1}\right|,\left|\ddot{\sigma}_{1}\right|\right) \leq\left(1.0 \cdot 10^{-6}, 1.3 \cdot 10^{-4}, 9.5 \cdot 10^{-3}\right), \\
\left(\left|\sigma_{2}\right|,\left|\dot{\sigma}_{2}\right|\right) \leq\left(7.9 \cdot 10^{-5}, 3.9 \cdot 10^{-3}\right) \\
\left(\left|\sigma_{1}\right|,\left|\dot{\sigma}_{1}\right|,\left|\ddot{\sigma}_{1}\right|\right) \leq\left(8.2 \cdot 10^{-9}, 9.9 \cdot 10^{-6}, 1.0 \cdot 10^{-3}\right) \\
\left(\left|\sigma_{2}\right|,\left|\dot{\sigma}_{2}\right|\right) \leq\left(9.8 \cdot 10^{-7}, 4.8 \cdot 10^{-4}\right)
\end{array}\right\} \text { for } \tau=10^{-4}
$$

which fits the standard homogeneous asymptotics $\sigma_{i}^{(j)}=O\left(\tau^{\operatorname{deg} \sigma_{i}^{(j)}}\right)[27]$ obtained for $\operatorname{deg} t=$ 1, i.e. $\sigma_{1}^{(j)}=O\left(\tau^{3-j}\right), j=0,1,2 ; \sigma_{2}^{(j)}=O\left(\tau^{2-j}\right), j=0,1$. 


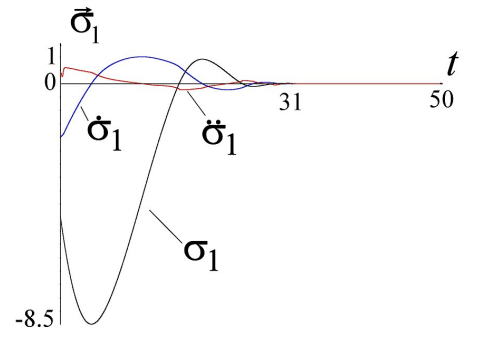

a.

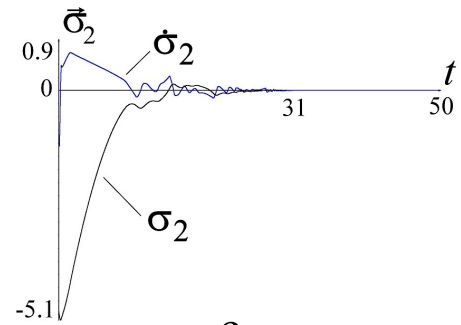

c.

no acceleration

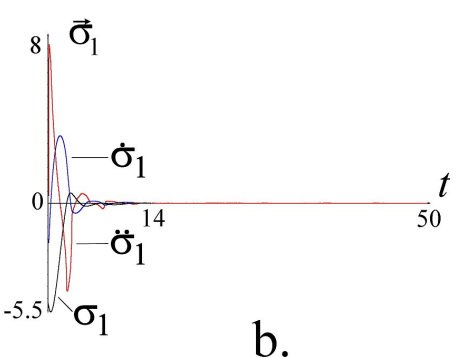

b.

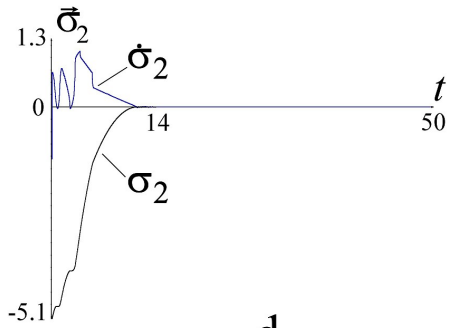

d.

\section{acceleration}

Figure 7.3: Comparison of the not accelerated (on the left) and accelerated (on the right) $(3,2)$-SM car control: convergence of the tracking errors and their derivatives to zero.

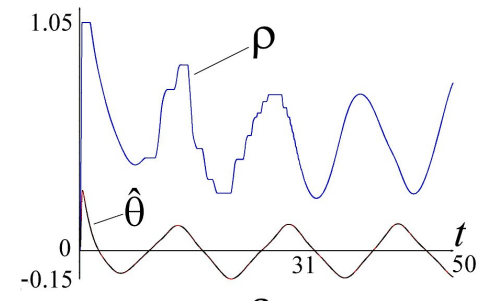

a.

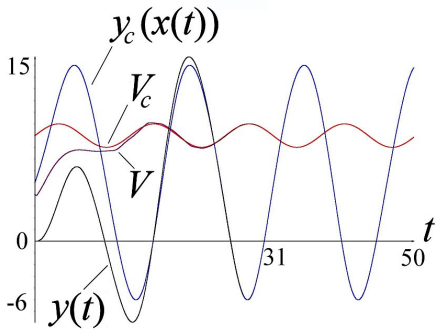

c. no acceleration

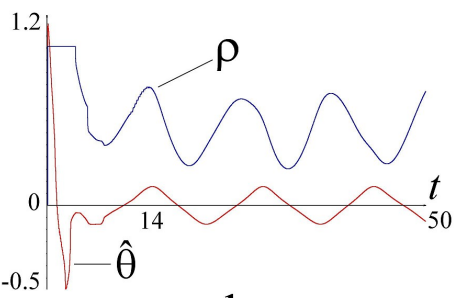

b.

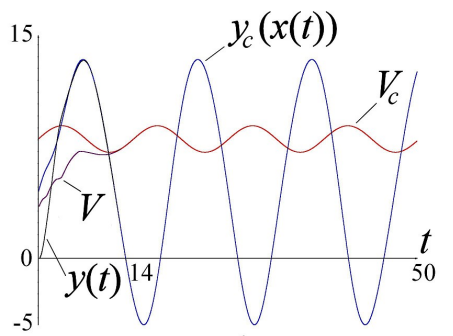

d.

Figure 7.4: Comparison of the not accelerated (on the left) and accelerated (on the right) $(3,2)$-SM car control. a,b: Actual controls, the gas throttle angle $\rho$ and the steering angle $\hat{\theta}$. c,d: Tracking performance.

Global fixed-time stabilization. Consider an academic MIMO control system

$$
\begin{aligned}
\left(\begin{array}{c}
\dddot{\sigma}_{1} \\
\dddot{\sigma}_{2}
\end{array}\right) & =\left(\begin{array}{c}
\sin \left(\sigma_{2}+\ddot{\sigma}_{2}\right)+\sin \left(\dot{\sigma}_{2}\right) \cos (5 t) \\
\left(\ddot{\sigma}_{1}+\sigma_{2}\right) /\left(1+\ddot{\sigma}_{1}^{2}+\sigma_{2}^{2}\right)
\end{array}\right)+ \\
& (2-\cos t)\left(\begin{array}{cc}
1+0.1 \cos \left(\dot{\sigma}_{1} \dot{\sigma}_{2}+\sigma_{1}+\sigma_{2}+2 t\right) & \cos t-0.1 \arctan \left(t+\ddot{\sigma}_{1}\right) \\
0.2 \cos \left(\dot{\sigma}_{2}+1.5 t+1\right) & 1-0.2 \sin (t+5)
\end{array}\right)\left(\begin{array}{l}
u_{1} \\
u_{2}
\end{array}\right) ;
\end{aligned}
$$

Copyright (c) 10000aळdhn) Wiley \& Spns, Ltd.

Int. J. Robust. Nonlinear Control (0000) Preparē using rncăuth. $\mathrm{ls} u=G^{\mathrm{s}} v$. 


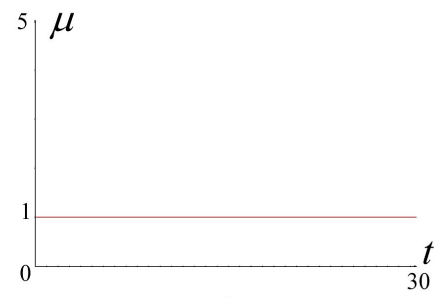

a.

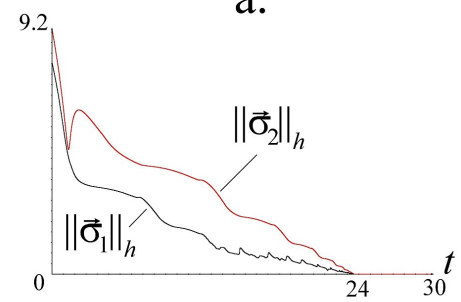

c.

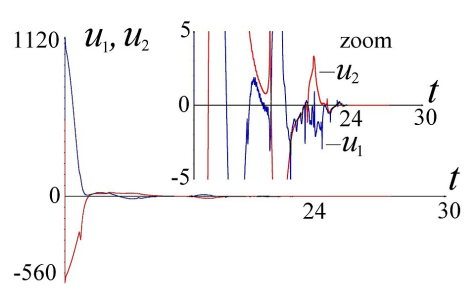

e.

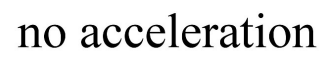

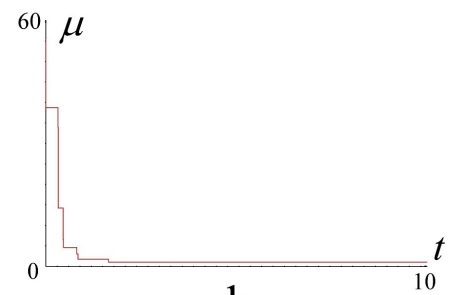

b.

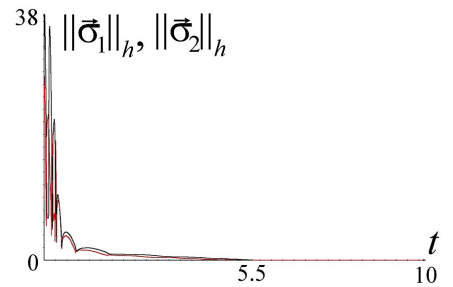

d.

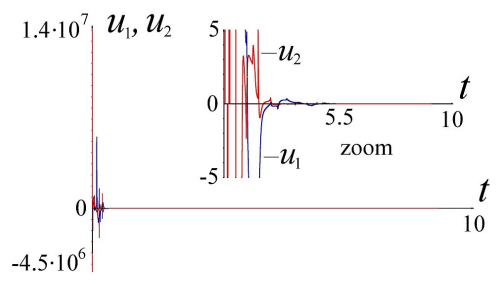

f.

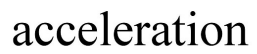

Figure 7.5: Stabilization of a system of the relative degree $(3,3)$ in $\mathbb{R}^{6}$ : not accelerated on the left and accelerated on the right. Acceleration factor $\mu$, homogeneous norms and controls.

The relative degree of the system is $r=(3,3)$. Let the system homogeneity degree be $-1, q=$ 2, the norms (6.4) be used with $\bar{\rho}_{1}=\bar{\rho}_{2}=3$. Thus, $\operatorname{deg} \sigma_{1}=\operatorname{deg} \sigma_{2}=5, \operatorname{deg} \dot{\sigma}_{1}=\operatorname{deg} \dot{\sigma}_{2}=4$, $\operatorname{deg} \ddot{\sigma}_{1}=\operatorname{deg} \ddot{\sigma}_{2}=3$. The accelerated controller of the family [28] is applied,

$$
\begin{aligned}
& v_{i}=-7 \mu^{3} N\left(\Lambda_{\mu} \vec{\sigma}\right) \cdot \Theta_{0.5}\left(\frac{\| \Lambda_{\mu} \vec{\sigma}_{i}||_{h}}{\| \Lambda_{\mu} \vec{\sigma}|| h}\right) \text { sat }\left[2 \frac{\ddot{\sigma}_{i} \mu^{-2}+2\left(\left|\dot{\sigma}_{i}\right| \mu^{-1}+\left|\sigma_{i}\right| \frac{4}{5}\right)^{-\frac{1}{4}}\left(\dot{\sigma}_{i} \mu^{-1}+\left\lfloor\sigma_{i}\right\rceil^{\frac{4}{5}}\right)}{\left|\ddot{\sigma}_{i}\right| \mu^{-2}+2\left(\left|\dot{\sigma}_{i}\right| \mu^{-1}+\left|\sigma_{i}\right|^{\frac{4}{5}}\right)^{\frac{3}{4}}}\right], \\
& N\left(\Lambda_{\mu} \vec{\sigma}\right)=\max \left(N_{1}, N_{2}\right), N_{i}=\left(\left|\ddot{\sigma}_{i}\right| \mu^{-2}+2\left(\left|\dot{\sigma}_{i}\right| \mu^{-1}+\left|\sigma_{i}\right|^{\frac{4}{5}}\right)\right)^{\frac{2}{3}}, i=1,2 .
\end{aligned}
$$

The value $\mu=1$ corresponds to the unaccelerated stabilization. The initial values $\sigma_{1}(0)=$ $200, \dot{\sigma}_{1}(0)=300, \ddot{\sigma}_{1}(0)=-500$ and $\sigma_{2}(0)=60, \dot{\sigma}_{2}(0)=-70, \ddot{\sigma}_{2}(0)=800$ are chosen. The integration was performed by the Euler method.

The accuracies obtained for the Euler/sampling step $\tau=10^{-4}, 10^{-5}$ are described by the component-wise inequalities

$$
\left.\begin{array}{l}
\left(\left|\sigma_{1}\right|,\left|\dot{\sigma}_{1}\right|,\left|\ddot{\sigma}_{1}\right|\right) \leq\left(5.6 \cdot 10^{-16}, 2.4 \cdot 10^{-12}, 2.5 \cdot 10^{-8}\right), \\
\left(\left|\sigma_{2}\right|,\left|\dot{\sigma}_{2}\right|,\left|\ddot{\sigma}_{2}\right|\right) \leq\left(2.1 \cdot 10^{-16}, 1.5 \cdot 10^{-12}, 1.6 \cdot 10^{-8}\right) \\
\left(\left|\sigma_{1}\right|,\left|\dot{\sigma}_{1}\right|,\left|\ddot{\sigma}_{1}\right|\right) \leq\left(5.6 \cdot 10^{-21}, 2.4 \cdot 10^{-16}, 2.5 \cdot 10^{-11}\right) \\
\left(\left|\sigma_{2}\right|,\left|\dot{\sigma}_{2}\right|,\left|\ddot{\sigma}_{2}\right|\right) \leq\left(2.1 \cdot 10^{-21}, 1.5 \cdot 10^{-16}, 1.6 \cdot 10^{-11}\right)
\end{array}\right\} \text { for } \tau=10^{-4}
$$


which perfectly fit the standard homogeneous asymptotics $\sigma_{i}^{(j)}=O\left(\tau^{\operatorname{deg} \sigma_{i}^{(j)}}\right)=O\left(\tau^{5-j}\right)[27]$ obtained for the system homogeneity degree -1 , i.e. for $\operatorname{deg} t=1$.

\section{CONCLUSIONS}

A simple acceleration method is proposed for the homogeneous FT stabilization of interconnected multiple chains of power integrators. The method utilizes a piece-wise-constant acceleration factor and is further applied to FT outputs' stabilization of uncertain nonlinear MIMO systems. Two main cases are studied: FT stabilization of some system outputs at zero, which corresponds to MIMO SM control; and FT stabilization of smooth MIMO systems at their equilibria by continuous control. In the latter case the outputs and their derivatives can serve as the system coordinates.

The remaining transient time along the trajectory is made to satisfy any prescribed upper estimation determined by a monotonous function (the convergence-rate function) of the stabilization-error homogeneous norm. No acceleration is allowed at the last convergence stage. Thus the final chattering is not amplified, and the accuracy of the original system [27, 35] is preserved in the presence of noises and discrete sampling. Fixed-time convergence is assured if the convergence-rate function is bounded.

Global continuity/quasi-continuity of the accelerated controls requires equal partial relative degrees. In practice the method is also applicable over compact operational regions or with artificially saturated acceleration factor. In such a case the requirement of equal partial relative degrees is removed.

The proposed controllers can be equipped with HOSM-based observers producing outputfeedback robust controllers. Such design is especially convenient in the case of SM control acceleration, since the upper control bound is an available piece-wise constant function of time.

References

1. I.M. Anan'evskii. Control synthesis for linear systems by methods of stability theory of motion. Differential Equations, 39(1):1-10, 2003.

2. D. Angeli and E.D. Sontag. Forward completeness, unboundedness observability, and their Lyapunov characterizations. Systems \&3 Control Letters, 38(4):209-217, 1999.

3. A. Bacciotti and L. Rosier. Liapunov Functions and Stability in Control Theory. Springer Verlag, London, 2005.

4. G. Bartolini, A. Pisano, E. Punta, and E. Usai. A survey of applications of second-order sliding mode control to mechanical systems. International Journal of Control, 76(9/10):875-892, 2003.

5. S.P. Bhat and D.S. Bernstein. Finite-time stability of continuous autonomous systems. SIAM Journal of Control and Optimization, 38(3):751-766, 2000.

6. S.P. Bhat and D.S. Bernstein. Geometric homogeneity with applications to finite-time stability. Mathematics of Control, Signals and Systems, 17(2):101-127, 2007.

7. I. Boiko and L. Fridman. Analysis of chattering in continuous sliding-mode controllers. IEEE Transactions on Automatic Control, 50(9):1442-1446, 2005.

8. F.H. Clarke, Y.S. Ledayev, and R.J. Stern. Asymptotic stability and smooth Lyapunov functions. Journal of Differential Equations, 149(1):69-114, 1998.

9. M. Defoort, T. Floquet, A. Kokosy, and W. Perruquetti. A novel higher order sliding mode control scheme. Systems \& Control Letters, 58(2):102-108, 2009.

10. S.H. Ding, A. Levant, and S.H. Li. Simple homogeneous sliding-mode controller. Automatica, 67(5):22-32, 2016.

11. F. Dinuzzo and A. Ferrara. Higher order sliding mode controllers with optimal reaching. IEEE Transactions on Automatic Control, 54(9):2126-2136, 2009.

12. H. Du and S. Li. Finite-time attitude stabilization for a spacecraft using homogeneous method. Journal of Guidance, Control and Dynamics, 35(3):740-748, 2012.

13. Y. Dvir and A. Levant. Accelerated twisting algorithm. IEEE Transactions on Automatic Control, 60(10):2803-2807, 2015.

14. C. Edwards and S.K. Spurgeon. Sliding Mode Control: Theory And Applications. Taylor \& Francis, 1998.

15. D. Efimov, A. Levant, A. Polyakov, and W. Perruquetti. Supervisory acceleration of convergence for homogeneous systems. International Journal of Control, published online 27 Dec 2016, 2017.

16. D. Efimov, A. Polyakov, A. Levant, and W. Perruquetti. Switched gain differentiator with fixed-time convergence. In Proc. 20th IFAC World Congress, Toulouse, July 9-14, 2017. 
17. A.F. Filippov. Differential Equations with Discontinuous Right-Hand Sides. Kluwer Academic Publishers, Dordrecht, 1988.

18. T. Floquet, J.P. Barbot, and W. Perruquetti. Higher-order sliding mode stabilization for a class of nonholonomic perturbed systems. Automatica, 39(6):1077-1083, 2003.

19. L. Fridman. Chattering analysis in sliding mode systems with inertial sensors. International Journal of Control, 76(9/10):906-912, 2003.

20. A.G. Gallardo Hernández, L. Fridman, A. Levant, Y. Shtessel, R. Leder, C. Revilla Monsalve, and S. Islas Andrade. High-order sliding-mode control for blood glucose: Practical relative degree approach. Control Engineering Practice, 21(5):747-758, 2013.

21. Y.G. Hong. Finite-time stabilization and stabilizability of a class of controllable systems. Systems 8 Control Letters, 46(4):231-236, 2002.

22. X.Q. Huang, W. Lin, and B. Yang. Global finite-time stabilization of a class of uncertain nonlinear systems. Automatica, 41(5):881-888, 2005.

23. A. Isidori. Nonlinear control systems I. Springer Verlag, New York, 1995.

24. P. Kaveh and Y.B. Shtessel. Blood glucose regulation using higher-order sliding mode control. International Journal of Robust and Nonlinear Control, 18(4-5):557-569, 2008.

25. M. Kawski. Homogeneous stabilizing feedback laws. Control Theory and Advanced Technology, 6:497-516, 1990.

26. A. Levant. Higher order sliding modes, differentiation and output-feedback control. International J. Control, 76(9/10):924-941, 2003.

27. A. Levant. Homogeneity approach to high-order sliding mode design. Automatica, 41(5):823-830, 2005.

28. A. Levant. Quasi-continuous high-order sliding-mode controllers. IEEE Trans. Aut. Control, 50(11):18121816,2005

29. A. Levant. Finite-time stabilization of uncertain SISO systems. In Proc. of the IEEE Conference on Decision and Control, CDC 2007, New Orleans, LA, USA, 2007.

30. A. Levant. Chattering analysis. IEEE Transactions on Automatic Control, 55(6):1380-1389, 2010.

31. A. Levant. Practical relative degree approach in sliding-mode control. Advances in Sliding Mode Control, Lecture Notes in Control and Information Sciences, 440:97-115, 2013.

32. A. Levant. Finite-time stabilization of uncertain MIMO systems. In Proc. of the 53th IEEE Conference on Decision and Control, Los-Angeles, December 15-18, 2014, 2014.

33. A. Levant, D. Efimov, A. Polyakov, and W. Perruquetti. Stability and robustness of homogeneous differential inclusions. In Proc. of the 55th IEEE Conference on Decision and Control, Las-Vegas, December 12-14, 2016, 2016.

34. A. Levant and M. Livne. Uncertain disturbances' attenuation by homogeneous MIMO sliding mode control and its discretization. IET Control Theory 83 Applications, 9(4):515-525, 2015.

35. A. Levant and M. Livne. Weighted homogeneity and robustness of sliding mode control. Automatica, 72(10):186-193, 2016.

36. A. Levant, M. Livne, and X. Yu. Sliding-mode-based differentiation and its application. In Proc. of the 20th IFAC World Congress, Toulouse, July 9-14, France, 2017, 2017, to appear.

37. A. Levant and Y. Pavlov. Generalized homogeneous quasi-continuous controllers. International Journal of Robust and Nonlinear Control, 18(4-5):385-398, 2008

38. W. Lin and C. Qian. Adding one power integrator: a tool for global stabilization of high-order lowertriangular systems. Systems \& Control Letters, 39(5):339-351, 2000.

39. J.A. Moreno and M. Osorio. Strict Lyapunov functions for the super-twisting algorithm. IEEE Transactions on Automatic Control, 57:1035-1040, 2012.

40. E. Moulay and W. Perruquetti. Finite-time stability and stabilization: state of the art. Lecture Notes in Control and Inf. Sciences, 334:23-41, 2006.

41. Y. Orlov. Finite time stability of switched systems. SIAM Journal of Control and Optimization, 43(4):1253-1271, 2005.

42. F. Plestan, A. Glumineau, and S. Laghrouche. A new algorithm for high-order sliding mode control. International Journal of Robust and Nonlinear Control, 18(4/5):441-453, 2008.

43. A. Polyakov. Nonlinear feedback design for fixed-time stabilization of linear control systems. IEEE Transactions on Automatic Control, 57(8):2106-2110, 2012.

44. A. Polyakov, D. Efimov, and W. Perruquetti. Finite-time and fixed-time stabilization: Implicit Lyapunov function approach. Automatica, 51:332-340, 2015.

45. A. Polyakov, D. Efimov, and W. Perruquetti. Robust stabilization of MIMO systems in finite/fixed time. International Journal of Robust and Nonlinear Control, 26(1):69-90, 2016.

46. R. Rajamani. Vehicle Dynamics and Control. Springer Verlag, 2005.

47. V.I. Utkin. Sliding Modes in Control and Optimization. Springer Verlag, Berlin, Germany, 1992.

48. S. Yu, X. Yu, B. Shirinzadeh, and Z. Man. Continuous finite-time control for robotic manipulators with terminal sliding mode. Automatica, 41(11):1957-1964, 2005.

49. D. Zhao, S. Li, Q. Zhu, and F. Gao. Robust finite-time control approach for robotic manipulators. IET control theory $E_{3}$ applications, 4(1):1-15, 2010. 